\title{
Extended Logic-plus-Functional Programming
}

Harold Boley

January 1992

Deutsches Forschungszentrum für Künstliche Intelligenz $\mathrm{GmbH}$

Postfach 2080

D-6750 Kaiserslautern, FRG

Tel.: (+49 631) 205-3211/13

Fax: (+49 631) 205-3210
Stuhlsatzenhausweg 3

D-6600 Saarbrücken 11, FRG

Tel.: (+49 681) 302-5252

Fax: (+49 681) 302-5341 


\section{Deutsches Forschungszentrum für Künstliche Intelligenz}

The German Research Center for Artificial Intelligence (Deutsches Forschungszentrum für Künstliche Intelligenz, DFKI) with sites in Kaiserslautern and Saarbrücken is a non-profit organization which was founded in 1988. The shareholder companies are Atlas Elektronik, Daimler-Benz, Fraunhofer Gesellschaft, GMD, IBM, Insiders, Mannesmann-Kienzle, SEMA Group, Siemens and SiemensNixdorf. Research projects conducted at the DFKI are funded by the German Ministry for Research and Technology, by the shareholder companies, or by other industrial contracts.

The DFKI conducts application-oriented basic research in the field of artificial intelligence and other related subfields of computer science. The overall goal is to construct systems with technical knowledge and common sense which - by using Al methods - implement a problem solution for a selected application area. Currently, there are the following research areas at the DFKI:

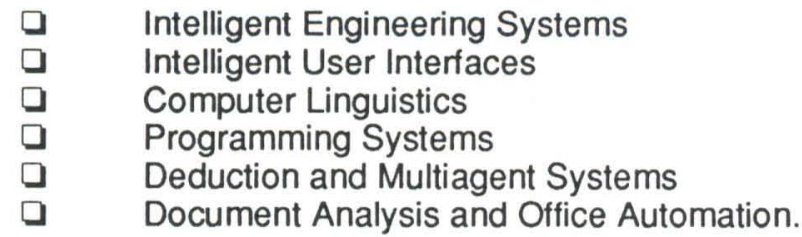

The DFKI strives at making its research results available to the scientific community. There exist many contacts to domestic and foreign research institutions, both in academy and industry. The DFKI hosts technology transfer workshops for shareholders and other interested groups in order to inform about the current state of research.

From its beginning, the DFKI has provided an attractive working environment for Al researchers from Germany and from all over the world. The goal is to have a staff of about 100 researchers at the end of the building-up phase.

Friedrich J. Wendl

Director 


\section{Extended Logic-plus-Functional Programming}

Harold Boley

DFKI-RR-92-03 


\section{To appear in:}

Lars-Henrik Eriksson, Lars Hallnäs, Peter Schroeder-Heister (eds.):

Proceedings of the second workshop on extensions of logic programming. ELP '91, SICS, Stockholm, Sweden, January 1991,

Springer, Lecture Notes in Artificial Intelligence, 1992.

This work has been supported by a grant from The Federal Ministry for Research and Technology (FKZ ITW-8902 C4).

This work may not be copied or reproduced in whole or in part for any commercial purpose. Permission to copy in whole or in part without payment of fee is granted for nonprofit educational and research purposes provided that all such whole or partial copies include the following: a notice that such copying is by permission of Deutsches Forschungszentrum für Künstliche Intelligenz, Kaiserslautern, Federal Republic of Germany; an acknowledgement of the authors and individual contributors to the work; all applicable portions of this copyright notice. Copying, reproducing, or republishing for any other purpose shall require a licence with payment of fee to Deutsches Forschungszentrum für Künstliche Intelligenz. 


\section{Contents}

1 Introduction 1

2 Relations Defined by Hornish Clauses 3

2.1 Open-World DATALOG . . . . . . . . . . . . . . . . . . 3

2.2 PROLOG-like Structures and Lists . . . . . . . . . . . . . . . 4

2.3 Varying-Arity Structures . . . . . . . . . . . . . . . . 5

2.4 Varying-Arity Relationships . . . . . . . . . . . . . . . 7

2.5 Higher-Order Constructors and Relations . . . . . . . . . . . . 8

3 Functions Defined by Footed Clauses 10

3.1 DATAFUN as a Functional Database Language . . . . . . . . . . . 10

3.1 .1 Footed Facts and Non-Ground Functions ............. 10 
3.1 .2 Footed Rules and the density Example . . . . . . . . . . . . 12

3.1.3 Non-Determinism, DATALOG Relationalizing, and WAM Compilation . . . . . . . . . . . . . . . . . . . 13

3.2 Full RELFUN Exemplified by "Self"-Functions . . . . . . . . . . . . 14

3.3 Higher-Order Constructors and Functions ............. 16

4 The Logic/Functional Style in Use

4.1 serialise: Inplace Updates of Non-Ground Structures . . . . . . . . 19

4.2 wang: On-the-Fly Construction of Proof Trees .......... 21

4.3 eval: Interpreting a LISP Subset in RELFUN ........... . . 23 


\title{
Extended Logic-plus-Functional Programming
}

\author{
Harold Boley \\ Deutsches Forschungszentrum für Künstliche Intelligenz \\ Box 2080, D-6750 Kaiserslautern, F. R. Germany \\ boley@informatik.uni-kl.de
}

\begin{abstract}
Extensions of logic and functional programming are integrated in RELFUN. Its valued clauses comprise Horn clauses ('true'-valued) and clauses with a distinguished 'foot' premise (returning arbitrary values). Both the logic and functional components permit LISP-like varying-arity and higher-order operators. The DATAFUN sublanguage of the functional component is shown to be preferable to relational encodings of functions in DATALOG. RELFUN permits non-ground, non-deterministic functions, hence certain functions can be inverted using an 'is'-primitive generalizing that of PROLOG. For function nestings a strict call-by-value strategy is employed. The reduction of these extensions to a relational sublanguage is discussed and their WAM compilation is sketched. Three examples ('serialise', 'wang', and 'eval') demonstrate the relational/functional style in use. The list expressions of RELFUN's LISP implementation are presented in an extended PROLOG-like syntax.
\end{abstract}

\section{Introduction}

Many approaches are possible for combining logic and functional programming, as illustrated by the collection [DL86]. These can be preclassified in two principal dimensions. (1) The combination may start with a model-theoretic semantics which is then refined (via proof theory) for practical programming or, it may start with an implemented operational semantics which is tuned in practice and then abstracted for model-theoretic foundation. (2) A quite separate distinction is whether one is interested in a loosly coupled hybrid system or, whether one strives for a tightly integrated logic/functional language.

With RELFUN we have been pursuing the latter alternatives of these dimensions: it is an operationally defined, highly integrated language (cf. [Bol86]). 
The language's operational spirit stems from its origin as a pure-LISP-based interpreter. Also the present version is both implemented in, and can access precoded functionality from (a subset of) COMMON LISP. Besides the definitional interpreter this implementation consists of a WAM compiler/emulator system. The RELFUN-in-LISP implementation runs all the examples to be presented here, where the speed is acceptable except, understandably, for the LISP-in-RELFUN example.

RELFUN's integrating concept is valued clauses, encompassing both PROLOG-style Horn clauses (for defining relations) and directed conditional equations (for defining functions). While the former start off from Horn logic, the idea for the latter is to regard a function definition like

$$
\operatorname{signum}(x)=\left\{\begin{array}{rll}
-1 & \text { if } & x<0 \\
0 & \text { if } & x=0 \\
1 & \text { if } & x>0
\end{array}\right.
$$

not as clauses of a logic with equality (shown on the left) but as clauses that return the right-hand sides of the directed equations via a ("\&"-marked) premise following after possible conditions (shown on the right):

eq $(\operatorname{signum}(X),-1):-X<0$.
eq $(\operatorname{signum}(0), 0)$.
eq $(\operatorname{signum}(X), 1):-X>0$.

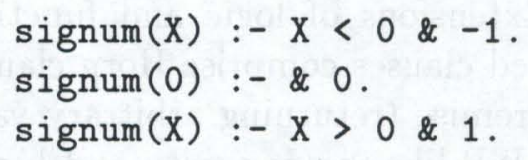

Hence, function calls need not be embedded into eq calls with auxiliary request variables, as in eq(signum(-2.7), SignumA), eq(signum(3.1), SignumB), SignumA < SignumB, but can be written directly, as in signum $(-2.7)<$ signum(3.1). We then interpret value-returning premises (after the ampersand) as generalized Horn-rule premises: apart from being terms like -1 they may be calls like $*(-1, X)$ or member $(X,[-1,-3,-5])$ and nestings likes $+(*(-1, X), 3)$ or member $(X$, rest $([-1,-3,-5]))$. Nestings are evaluated strictly call-by-value, as, classically, in FP [Bac78].

The RELFUN notions of relation and function are amalgamated to an abstract operator concept: functions are generalized to non-ground, non-deterministic operators, hence relations can be viewed as characteristic functions. Our notion of relations as true-valued functions is like in SLOG [Fri85], except that RELFUN's valued facts return true implicitly. Another amalgamating notion is akin to LISP's "useful non-nil values": relationlike operators may on success return a value more informative than true (e.g., we can let member return the list starting from the element found). All kinds of RELFUN operators can be applied in generalized Horn-rule premises, which are usable uniformly to the left as well as to the right of the "\&"-separator. Actually, such premises constitute a valued conjunction, also permitted as a top-level query (e.g., member $(\mathrm{X}, \mathrm{L})$ \& member $(\mathrm{X}, \mathrm{M})$ non-deterministically returns rest lists of $M$ whose first element also occurs in $L$ ). A special valued conjunction calling only relations to the left of " $\&$ " and having a single variable to its right (e.g., country $(X)$, between $(X$, atlantic, pacific) \& $X)$ can be viewed as an indefinite description or $\eta$-expression (e.g., $\eta(x)[$ country $(x) \wedge$ between (x, atlantic, pacific)] , also provided in other relational/functional amalgamations (see [PS91]).

Certain RELFUN functions can be inverted by calling them non-ground (by-value) on the right-hand side (rhs) of a generalized PROLOG is-primitive, mimicking relations 
(incl. the above eq predicate). RELFUN thus provides a version of innermost conditional narowing [Fri85]. Its operational semantics flattens functional nestings to relational conjunctions; thus inherits the search-space reduction of SLD-resolution [BGM88]. Hence, our WAM implementation of (first-order) RELFUN can approach the speed of PROLOG [Bol90].

Besides its attempt at integrating basic notions of PROLOG and LISP, many of RELFUN's extended concepts can also be transferred to relational and functional programming individually. In the following section (2) the extended relational component will be treated, including higher-order relations. The next section (3) will then augment this by the extended functional component and discuss its benefits. Finally, the section (4) before the conclusions will give three sample uses of the relational/functional style.

\section{Relations Defined by Hornish Clauses}

\subsection{Open-World DATALOG}

First we consider DATALOG i.e., PROLOG without structures (constructor symbols applied to arguments). This kernel language of deductive databases is also a subset of RELFUN. DATALOG clauses have identical syntax ${ }^{1}$ and equivalent semantics in PROLOG and RELFUN. Queries to RELFUN differ only as follows: they return the truth-value true instead of printing the answer yes; they signal failure by yielding the truth-value unknown instead of printing no.

When we stay in the relational realm of RELFUN this makes not much of a difference since true can be mapped to yes and unknown can be mapped to no. However, when proceeding to RELFUN's functional realm, queries will be able to return the third truthvalue false: this is to be mapped to those of PROLOG's no answers for which the closed-world assumption is justified. In general, however, RELFUN does not make the closed-world assumption, and in the absence of explicit negative information modestly yields unknown instead of 'omnisciently' answering no.

For example, given the DATALOG knowledge base

subfield (architecture, bridgebuilding).

applicable (pharmacy, medicine).

applicable (computerscience, bridgebuilding) .

applicable (computerscience, computerscience).

applicable(Tool,Field) :- subfield(Field,Sub), applicable(Tool,Sub).

a successful query like applicable(computerscience,architecture) returns true in RELFUN and prints yes in PROLOG; however, a failing query like

\footnotetext{
${ }^{1}$ The syntax shown for full RELFUN will continue to be PROLOG-like. In the implementation it becomes equivalent LISP-like list expressions. Although the (older) LISP-like syntax will not be shown in this paper, it is actually used more often than the (newer) PROLOG-like syntax. RELFUN has been given two syntaxes to facilitate communication between users from the LISP and PROLOG communities.
} 
applicable (computerscience, agriculture) yields unknown in RELFUN but prints no in PROLOG. As with most real-life knowledge, what we know about computer-science applications is inherently open-ended; RELFUN's unknown reply agrees to the required open-world semantics.

Later, in DATAFUN, certain relations such as subfield will be reformulated as functions (cf. subsection 3.1). This will also have consequences for 'Horn' rules such as the applicable rule which still define a relation but call a subfunction, e.g., in an is-rhs: applicable(Tool,Field) :- Sub is subfield(Field), applicable(Tool,Sub). To accomodate such functional (and is-'equational') extensions in relational rules, we speak of hornish rules or, generally, hornish clauses.

Two further extensions of DATALOG, varying-arity DATALOG and higher-order DATALOG, will be treated implicitly in the corresponding full-PROLOG extensions (see subsections 2.4 and 2.5 ).

\subsection{PROLOG-like Structures and Lists}

Let us now proceed to PROLOG with structures and its RELFUN extensions. PROLOG has only constructor symbols and no defined function symbols; arguments to PROLOG relations must always be (passive) structures and can never be (active) calls. RELFUN, on the other hand, does support both of these categories, hence has a notational need to distinguish between them.

First consider the more basic distinction of relations on the one hand, and constructors and defined functions on the other hand: while mathematical accounts of first-order logic express the distinction by disjoint sets of relation (predicate) and function symbols, PROLOG just distinguishes predicate (top-level) and functor (sublevel) uses of these symbols, and permits the same symbol to occur as a predicate and as a functor. This permits metalogical reinterpretations of certain structures as goals (via call).

In the same interactive-programming spirit RELFUN does not distinguish active and passive functor symbols but just active and passive functor uses. For this we note that all functor uses take the form of applications, which we write with round parentheses for 'active' operator calls and with square brackets for 'passive' structured terms. In the relational part of RELFUN this means that only top-level relation calls are written with parentheses, PROLOG-like structures are written with brackets. (In the functional part both top-level and nested function calls will be parenthesized, too.)

Consider the successor constructor s, often used together with 0 for specifying invertible operations on natural numbers. Thus, while in PROLOG the structure corresponding to 2 is $\mathbf{s}(\mathbf{s}(0))$, in RELFUN it is $\mathbf{s}[\mathbf{s}[0]]$. For instance, the RELFUN lesseq relation definition

lesseq $(0, N)$.

$\operatorname{lesseq}(s[M], s[N]):-\operatorname{lesseq}(M, N)$.

permits the call lesseq $(\mathrm{X}, \mathrm{s}[\mathrm{s}[0]])$ to generate the $\mathrm{X}$-values $0, \mathbf{s}[0]$, or $\mathbf{s}[\mathbf{s}[0]]$. 
$\mathrm{N}$-element RELFUN lists, as in LISP and PROLOG, can be regarded as a short-hand for nested binary structures (we use the distinguished constructor "cns" instead of the usual ":"). For example, the (non-ground) list $[s[s[0]],[E, F], s[0]]$ reduces to the nesting $\mathrm{cns}[\mathrm{s}[\mathrm{s}[0]], \mathrm{cns}[\mathrm{cns}[\mathrm{E}, \mathrm{cns}[\mathrm{F}, \mathrm{nil}]], \mathrm{cns}[\mathrm{s}[0], \mathrm{nil}]]]$. A vertical bar in lists causes their cns-reduction to end with the element after the "I" (usually a variable) rather than with the distinguished constant nil. Thus, $[X, Y \mid Z]$ reduces to $\mathrm{cns}[\mathrm{X}, \mathrm{cns}[\mathrm{Y}, \mathrm{Z}]]$. This "lists-to-structures" transformation is used both for WAM compilation and mathematical formalization. Note that the sorted relation definition in PROLOG/RELFUN

sorted ([]).

$\operatorname{sorted}([\mathrm{X}])$.

$\operatorname{sorted}([X, Y \mid Z]):-\operatorname{lesseq}(X, Y)$, $\operatorname{sorted}([Y \mid Z])$.

and its cns-reduced form in RELFUN

sorted (nil).

$\operatorname{sorted}(\mathrm{cns}[\mathrm{X}, \mathrm{nil}])$.

$\operatorname{sorted}(\operatorname{cns}[X, \operatorname{cns}[Y, Z]]):-\operatorname{lesseq}(X, Y), \operatorname{sorted}(\operatorname{cns}[Y, Z])$.

consistently employ square brackets to indicate the 'passiveness' of lists and structures, while in PROLOG the cns-reduced form would employ round parentheses.

\subsection{Varying-Arity Structures}

Lists can also be given a direct $\mathrm{N}$-element interpretation because RELFUN permits varying-arity structures i.e., structures containing a vertical bar. Like cns was used as a binary list constructor we use tup as an $\mathrm{N}$-ary list constructor $(\mathrm{N} \geq 0)$. That is, [...] should be regarded as an abbreviation for tup [...]. This convention holds even if [...] contains a "I". So, the earlier lists really stand for $\operatorname{tup}[s[s[0]], \operatorname{tup}[E, F], s[0]]$ and $\operatorname{tup}[\mathrm{X}, \mathrm{Y} \mid \mathrm{Z}]$. Such tup structures can again be viewed as nested cns structures as shown for lists above.

Varying arities are also permitted for all other RELFUN constructors. This can be used for reinterprating many 'untyped' list representations as constructor-'tagged' structures. For instance, unbounded staples and dumps of elements can be written as varying-arity structures staple[...] and dump [...], whose constructors distinguish the two 'types' of element collections. The unification of RELFUN structures containing a "I" generates a list value for a variable after the "I", as if the "I" would appear in a list context. Similarly, lists constitute the only structures to be spliced into other structures after the "I". Lists are thus the "neutral' data structure for transporting the "I"-remainders of varying-arity structures.

For example, staple[book,folder,folder|Rest] represents a staple with a book followed by two folders on the top, and some unspecified remainder Rest. When unified with staple[book, Y, Y, paper, Z, paper], Y is bound to folder and Rest to the list 
[paper, $\mathrm{Z}$,paper]. This list can again be spliced into, say, a dump beginning with a book, dump [book|Rest], resulting in dump [book, paper, Z, paper].

Unlike PROLOG we permit the vertical bar to follow directly after an opening square bracket, both in lists and in (other) structures. For any list X, the list [IX] is the same as $\mathrm{X}$; additionally given a constructor $\mathrm{c}$, the structure $\mathrm{c}[\mathrm{IX}]$ exclusively uses the elements of the list $\mathrm{X}$ as its arguments. Thus with the Rest binding [paper, $\mathrm{Z}$,paper], dump [IRest] is equivalent to dump [paper, $Z$, paper].

It is now possible to define elementwise equality of staples and dumps using the facts

argumenteq (staple[|Args], dump[|Args]). argumenteq(dump [|Args], staple[|Args]).

where the two Args occurrences of each fact will be bound to unifying lists of elements. Thus, while staple[book,X,X] and dump[Y,paper,paper] would not unify, the call argumenteq (staple [book, X, X], dump $[\mathrm{Y}$, paper, paper]) succeeds.

Another use of varying-arity structures is the term representation of clauses themselves. In PROLOG ":-" can be regarded as a binary functor whose arguments are the clause head and a nesting of binary ", ," "-conjunctions for the body; in RELFUN it is reinterpreted more concisely as an $\mathrm{N}$-ary constructor $(\mathrm{N} \geq 1)$ whose first argument is the head and whose remaining arguments make up the body conjuncts. The rule of the DATALOG example in subsection 2.1 thus becomes the PROLOG structure

:- (applicable(Tool,Field),

', '(subfield(Field, Subfield), applicable(Tool, Subfield)))

and the RELFUN structure

:-[applicable[Tool,Field], subfield[Field,Subfield] , applicable[Tool, Subfield]]

The use of lists to treat "I" in all contexts suggests a technique for reducing varyingarity structures to fixed-arity ones. Each varying-arity $c[\mathrm{x} 1, \ldots, \mathrm{xN} \mid \mathrm{X}]$ could be replaced by the unary $c[[\mathrm{x} 1, \ldots, \mathrm{xN} \mid \mathrm{X}]]$, where the single argument is a list containing the original c arguments as elements. However, this naive method introduces unnecessary bracketing (which could be hidden to the user) and hinders intrastructure WAM indexing [Sin92] with respect to a structure's top-level arguments (which become 'neutralized' to a tup or cns constructor). Instead of listifying all $c$ arguments, a 'semi-listifying' method might keep a fixed number, $\mathrm{K} \leq \mathrm{N}$, of initial arguments and only listify the remaining ones, resulting in the $(\mathrm{K}+1)$-ary $c[\mathrm{x} 1, \ldots, \mathrm{xK},[\mathrm{xK}+1, \ldots, \mathrm{xN} \mid \mathrm{X}]]$. However, even if global static analysis is used to find the smallest $\mathrm{K}$ such that a vertical bar or a closing square bracket is used after the Kth argument of $c$ (for $\mathrm{K}=0$ leading back to the naive method), an interactive user could employ $c[a 1, \ldots, a I \mid R]$ with $I<K$. In certain queries such a structure could be pretranslated to $c[a 1, \ldots, a I, R 1, \ldots, R J, R *]$, with $I+J=K$, by "unrolling' the variable $R$ used after the "I" i.e., generating new variables $R 1, \ldots, R J$ and $R *$, and on success binding $R$ to $[R 1, \ldots, R J \mid R *]$. In general, it is hard to avoid making possible query patterns statically known to the global analyzer. 


\subsection{Varying-Arity Relationships}

Proceeding from constructor terms to atomic formulas, we come to the LISP-inspired PROLOG extension of varying-arity relation applications i.e., clause heads and bodies directly containing a "I". Thus, both structures and applications can be ended by a vertical bar followed by an ordinary variable; equivalently, they could be ended by a "sequence variable" as used in KIF [GF91]. Varying-arity applications give argument sequences the flavor of an implicit list data structure. For instance, the $\mathrm{N}$-ary version ( $\mathrm{N}$ $\geq 0$ ) of the sorted relation

sorted ().

sorted $(X)$.

$\operatorname{sorted}(X, Y \mid Z):-\operatorname{lesseq}(X, Y)$, $\operatorname{sorted}(Y \mid Z)$.

permits calls like sorted $(0, \mathrm{~W}, \mathrm{~s}[\mathrm{~s}[0]], \mathrm{s}[\mathrm{s}[\mathrm{s}[0]]])$, binding $\mathrm{W}$ to $0, \mathrm{~s}[0]$, or $\mathrm{s}[\mathrm{s}[0]]$.

As in LISP, the $\mathrm{N}$-ary flexibility gained can be used, among other things, to flatten nestings of binary associative operators like + and append. Their output cannot go to the (usual) last argument position because of the asymmetry of "I"-list-splicing; the only uniformly usable output argument is the first one.

For example, while ordinary PROLOGs' ternary append relation is already quite flexible, LM-PROLOG [CK85] defines a natural $\mathrm{N}$-ary extension $(\mathrm{N}>0)$, which in RELFUN is rewritten as

append ([]).

append(Total, []|Back) :- append(Total|Back).

append([First|Total], [First|Front]|Back) :- append(Total, Front|Back).

It 'contains' LISP's unary null predicate, a list-typed PROLOG-like binary "=" relation, and a permuted, list-typed version of PROLOG's ternary append relation (append $(1,[], 1)$ won't succeed), but is actually a varying-arity relation, which can be used in surprisingly diverse ways. Two samples are append([a,b,c],L1,..,Lm), splitting a given list into arbitrarily many lists, and append ( $[\mathrm{a}, \mathrm{b}, \mathrm{a}, \mathrm{b}, \mathrm{a}, \mathrm{b}]$, Leftcontext, $[\mathrm{a}, \mathrm{b}, \mathrm{a}]$, Rightcontext), unifying symmetric list segments.

Of course, a simple transformer can put the varying number of arguments of such relations into a single list. For sorted the additional brackets would lead back to the original definition; for append, with its distinguished first argument, however, they would become a syntactic burden. Also, the transformation can result in serious problems for even the standard WAM-indexing scheme because the first (and only) relation argument becomes of type list indiscriminately. This could be remedied by a version of the semilistifying arity-fixing technique sketched for structures in subsection 2.3 (e.g., listifying only the $\mathrm{N}-1$ input lists of append). 


\subsection{Higher-Order Constructors and Relations}

While PROLOG restricts constructors and relations to constants, RELFUN also permits them to be variables or structures. This enables a restricted kind of higher-order operators, syntactically reducible to first-order operators, but more expressive and cleaner than PROLOG's use of extralogical builtins like functor, "=. .", and metacall as higher-order substitutes. Higher-order unification of the kind studied with $\lambda$ Prolog [NM90], however, is orthogonal to the extensions in RELFUN, which for simplicity and efficiency lives without $\lambda$-expressions (thus avoiding problems with $\lambda$-variables [Bac78]) and 'semantic' extensions of Robinson unification.

Constructor variables can be used to abstract from, or force equality of, the 'type' of structures, as encoded by their constructor. For example, the unification of staple[book, X,X] and $\mathrm{F}[\mathrm{Y}$,paper,paper] succeeds, binding $\mathrm{F}$ to the constructor staple. Also, the argumenteq definition of subsection 2.3 can be generalized to arbitrary constructors, using a single fact:

argumenteq(F[|Args $], G[\mid$ Args $])$.

A converse definition, of constructoreq,

constructoreq(F[|Args1], F[IArgs2]).

may be used to check equality of only the 'types' of two structures, as in the successful constructoreq (staple[paper,book], staple[book, folder, X]). For PROLOG's structures constructoreq could be simulated by two calls of the functor builtin.

Constructor structures embody parameterized constructors such as stack [integer], which are themselves applicable to arguments as in stack [integer] $[3,1,2]$. The above constructoreq fact can thus be refined to a conspareq definition, succeeding for equally parameterized constructor applications such as a stack and a heap of integers:

conspareq(F [Argtype] [IArgs1], G[Argtype] [|Args2]).

The variables $\mathrm{F}$ and $\mathrm{G}$ stand here for constructors, e.g. stack and heap, of constructor structures, whose single Argtype parameters must be equal.

Relation variables in queries enable to find all relationships between given arguments. In the DATALOG knowledge base (cf. subsection 2.1) the query $\mathrm{R}$ ( $\mathrm{X}$, bridgebuilding) needs only fact retrieval for binding $\mathrm{R}$ to the relation subfield and $\mathrm{X}$ to the object architecture or, $\mathrm{R}$ to applicable and $\mathrm{X}$ to computerscience; the query $\mathrm{R}$ (computerscience,architecture) requires rule deduction for binding $\mathrm{R}$ to applicable. Later, using footed clauses (section 3), relations found in this way will become returnable values, as in $R(X, X)$ \& self $[R][X]$, returning self [applicable] [computerscience], where the R-value is part of a constructor structure. Note that the R's employed here are 'relation-request' variables, free at the time of invoking the queries. More usual (mainly in LISP-based PROLOGs) is to permit variables 
in relation position only if they are always bound at the time of the call, as in the example of the next paragraph.

Relation variables in clauses permit the use of higher-order facts (recognized as such by the context) like virtue(supports), virtue (protects), etc. to abstract rules like

honorable(X) :- $\operatorname{supports}(X, Y)$.

honorable(X) :- $\operatorname{protects}(X, Y)$.

etc. to the single rule ("Honorable is who has a virtuous relationship to someone")

honorable(X) :- virtue(R), R(X,Y).

Here we apply virtue as a unary second-order relation over binary relations, but more general higher-order relations can be useful.

Relation structures can be employed for defining operations on relations. For example, the relational product can be defined using the structure relproduct $[R, S]$ as a relation, which permits relational square to be defined with just a fact that uses a relproduct structure as its second argument:

relproduct $[R, S](X, Z):-S(X, Y), R(Y, Z)$.

relsquare $(R$, relproduct $[R, R])$.

While the structure relproduct[...] can be (higher-order-)called directly, as in relproduct [fathrel, mothrel] ( $j \circ \mathrm{hn}, \mathrm{W}$ ), the constant relsquare is (first-order-)called to bind a variable, which is then used as a structure-valued relation variable, as in relsquare(fathrel, T), T(john,W).

As discussed in [Bol90], higher-order relations of this form are not easily compiled into the WAM, which collects all clauses with the same constant relation name and arity into a procedure. However, relation variables and structures can be eliminated by simply introducing an apply relation constant as in [War82], which we shorten to ap: hor (...) is replaced by ap (hor, ...) in all heads and bodies, moving the higher-order relation hor to the first argument position. The last example thus becomes

$\operatorname{ap}(\operatorname{relproduct}[R, S], X, Z):-\operatorname{ap}(S, X, Y), \operatorname{ap}(R, Y, Z)$.

ap (relsquare, $R$, relproduct $[R, R]$ ).

and can be queried by, e.g., ap(relsquare,father, $T$ ), ap $(T, j \circ h n, W)$. Note that the relsquare clause and goal would not have needed the ap dummy because the relsquare relation is a constant. However, even if all calls to a relation in a program can be found to be first-order by static analysis, the user could still issue relationvariable queries like $P(R$, relproduct $[R, R])$. In the WAM these would only work in the form $\operatorname{ap}(P, R, \operatorname{relproduct}[R, R])$, and presuppose that the relsquare clauses are aptransformed, like all other ones. Consider the effect of having all clauses collected into ap/i procedures, whose first arguments always are the former relation names (hence, $i>$ 
0 ). The discriminating effect of calling differently named procedures is lost, but is simulated by the usual first-argument indexing, loosing of course the refined discrimination of non-ap first-argument indexing. Fortunately, in our WAM we can index on all arguments (to the left of "I"), thus regaining full discriminative power for ap-reduced clauses.

For constructor variables and structures an analogous first-order reduction is possible using a dummy constructor, which should again be ap in order to permit metacalls for reduced clauses. As for earlier reductions this will affect WAM indexing: (top-level) structure's constructors are all mapped to the same dummy constant, loosing the constructors' indexing power, which could be regained by also indexing on their first arguments.

\section{$3 \quad$ Functions Defined by Footed Clauses}

\subsection{DATAFUN as a Functional Database Language}

We now proceed to functions, first considering DATAFUN, the functional subset of RELFUN corresponding to PROLOG's DATALOG subset.

\subsubsection{Footed Facts and Non-Ground Functions}

Let us consider the database example in [WPP77], containing the following DATALOG facts about country areas (given in thousands of square miles):

area(china, 3380).
area(india, 1139).
area(ussr, 8708).
area(usa, 3609).

Although these binary relations would permit requests like area (Cntry, 8708), their normal use direction is of the kind area(ussr, Area): the large value range of possible areas makes it unlikely that a user ask for a country with a precisely given thousands-of-squaremiles area such as 8708 (the problem would become even more noticeable if the exact areas were stored, perhaps as real numbers, with rounding problems etc.) Therefore ${ }^{2}$, in our opinion this 'historical' DATALOG example should be rewritten functionally, as already implied in [GM84]. For this we extract the second argument from the DATALOG facts and use it as the so-called foot after a ": -\&"-infix:

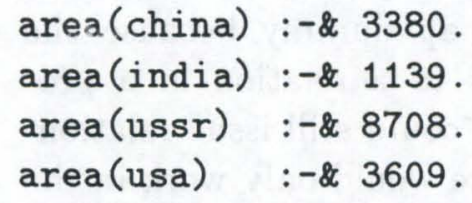

\footnotetext{
${ }^{2}$ We do not make use of the argument that area $:$ Cntry $\longrightarrow$ Area is a mapping (or 'functional' in, e.g., the relational database sense) while its inverse is not (some small countries' areas coincide if rounded to 1000 sq. mi.), because RELFUN does allow non-deterministic functions, as will be shown shortly.
} 
The resulting special DATAFUN clauses are called footed facts, here used for the pointwise definition of the RELFUN function area mapping from country names to natural numbers. The definition emphasizes the natural area use direction, as in area(ussr), a function call returning the value 8708 .

The main advantage of distinguishing an 'output' argument of a relation as the returned value of a corresponding function is the possibility of nested calls such as

$+(\operatorname{area}($ china $), \operatorname{area}($ india $), \operatorname{area}($ usa) $)$

where the parenthesized inner applications are (not passive structures but) active function calls that return their values to the ternary + use (cf. subsection 2.2); for reasons of conciseness, program analysis, and variable elimination this is preferable to flat relational conjunctions such as

$\operatorname{area}($ china, $A 1)$, area(india, $A 2), \operatorname{area}($ usa,$A 3),+($ Area, $A 1, A 2, A 3)$

The main disadvantage lies in the issue of inverted calls, which are easier and sometimes more logically complete for 'usage-neutral' relations: a functional non-termination problem is illustrated in [Fri84]. However, RELFUN's inversion method for functions appears quite natural, and for its DATAFUN subset completeness problems do not arise. A generalized form of PROLOG's is-primitive is employed to unify the values of a free function call with the value to be used as the argument of the inverse function, where a call is free if all its (actual!) arguments are different free variables. More generally, DATAFUN (RELFUN) permits non-ground function calls which like DATALOG (PROLOG) goals may contain repeated logical variables (non-ground terms).

As a simple example with just one free variable consider 8708 is area(Cntry), the inverse function call corresponding to the above-discussed relational inversion area(Cntry, 8708). Independently from the context (e.g., in an is-rhs) the free call area(Cntry) non-deterministically returns the values $3380,1139,8708$, or 3609 , at the same time binding Cntry to china, india, ussr, or usa, respectively, in the textual order of the area footed facts in the knowledge base. Within the above is-call only the third of the returned values unifies with the left-hand side (lhs), so the inversion correctly binds Cntry to ussr.

Other operators such as the exponentiation relation may be hardly or impossibly inverted, which again suggests to rewrite them as 'directed' functions, leading from nonground facts like

$\exp (X, 0,1)$.

$\exp (X, 1, X)$.

to non-ground footed facts like

$\exp (X, 0):-\& 1$

$\exp (X, 1):-\& X$. 
Here, the first clause has a ground foot, 1, while the second one has a non-ground foot, $X$ (in DATAFUN this must be a variable). Non-ground foots can yield both ground and non-ground values, as in $\exp (2,1)$, returning 2 , and $\exp (Y, 1)$, returning $Y$, respectively.

\subsubsection{Footed Rules and the density Example}

In [WPP77] there are also DATALOG Horn facts about population (in millions), which we think should be 'functionalized' to DATAFUN footed facts as demonstrated for area. On this basis the paper supplies the population density (per square mile) of a country, using the DATALOG rule (somewhat extralogical because of the is-call for D)

$\operatorname{density}(C, D):-\operatorname{pop}(C, P), \operatorname{area}(C, A), D$ is $(P * 1000) / A$.

This can be mimicked by the equivalent DATAFUN rule (with is-calls for $\mathrm{P}$ and $\mathrm{A})^{3}$

$\operatorname{density}(C):-\quad P$ is $\operatorname{pop}(C), A$ is $\operatorname{area}(C)$ \& $/(*(P, 1000), A)$.

which may be condensed to the DATAFUN rule (without is-calls or auxiliary variables)

$\operatorname{density}(C):-\& \quad /(*(\operatorname{pop}(C), 1000)$, area(C)).

Rules containing an "\&" separator are called footed rules. The rule premises to the left of " $\&$ " are called body premises and act exactly like the premises of a hornish rule. The premise to the right of " $\&$ " is called a foot premise and differs from the other premises only in that its value becomes the value of the entire rule. Together, these premises form a valued conjunction, which like an " $\&$ "-less conjunction can also be used directly as a query. Footed facts are special footed rules with an empty conjunction of body premises (the separator sequence ": - \&" is normally joined to ": $-\& ")$ and a foot premise which just denotes a value (without evaluation). So the shortened footed density rule above is not a footed fact since its foot evaluates an expression. The most natural use of the DATAFUN database would be functional calls like density (usa), returning the density value for usa. However, these rule formulations could also be inverted or even be called freely to enumerate all country/density pairs as in the relational call density (Cntry, Dnsty) (delivering both countries and their densities as bindings) or the functional call density (Cntry) (delivering countries as bindings with their densities as values).

To conclude the density example of [WPP77], PROLOG's "database query" rule

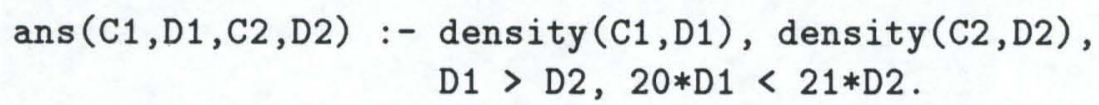

and request ans $(\mathrm{C} 1, \mathrm{D} 1, \mathrm{C} 2, \mathrm{D} 2)$ for finding countries whose population density differs by less than $5 \%$, in RELFUN could be mimicked directly but can also be rewritten as a single valued conjunction

\footnotetext{
${ }^{3}$ Following LISP, RELFUN currently does not distinguish arithmetic operators as infixes, but like all other operators applies them as prefixes.
} 
D1 is $\operatorname{density}(C 1), D 2$ is $\operatorname{density}(C 2),>(D 1, D 2),<(*(20, D 1), *(21, D 2))$ \&

ans $[\mathrm{C} 1, \mathrm{D} 1, \mathrm{C} 2, \mathrm{D} 2]$

where the auxiliary global ans relation transmutes to a temporary ans constructor.

\subsubsection{Non-Determinism, DATALOG Relationalizing, and WAM Compilation}

While free calls for the inversion of the area and density functions produce nondeterministic results, the area and density definitions themselves are deterministic. In RELFUN non-deterministic function definitions are also allowed, which return more than one value even for ground calls.

For instance, the subfield relation of the DATALOG example in subsection 2.1 could be extended non-deterministically, expanded by a transitive-closure version subclosure, and transcribed into a function definition, as in the following DATAFUN example:

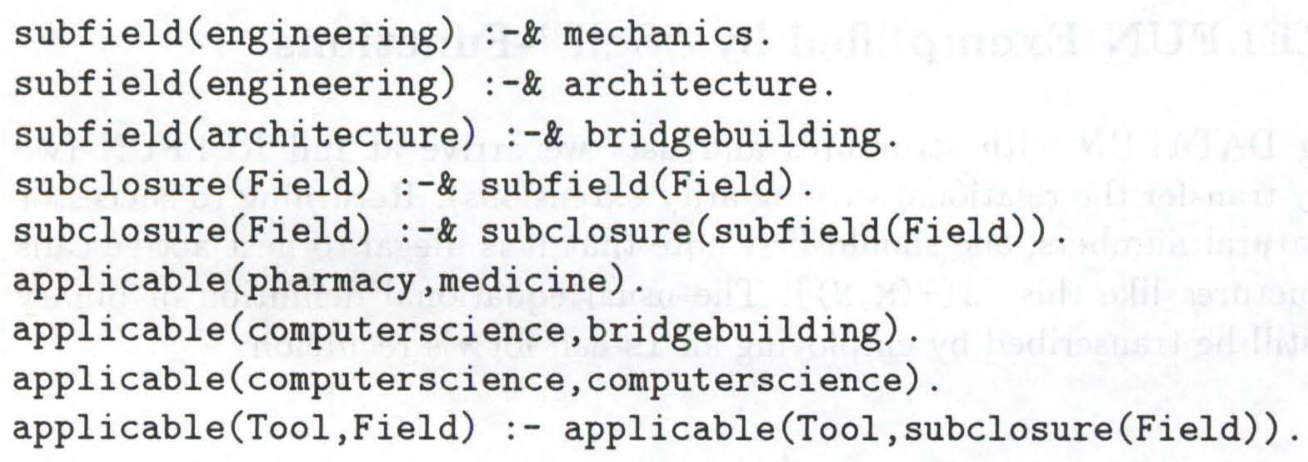

In this knowledge base the ground call subfield (engineering) non-deterministically returns the values mechanics or architecture; finding a subfield path from engineering to bridgebuilding, applicable(computerscience, engineering) returns true. Note that the operator applicable itself is left a relation but its former Horn rule using a flat relational conjunction became a hornish rule that nests the (non-deterministic!) subclosure function into the recursive call. The original relational form could again be mimicked using an is-call, leading to

applicable(Tool,Field) :- Sub is subclosure(Field), applicable(Tool,Sub).

This flattening of the applicable definition exemplifies the first step of RELFUN's relationalize transformation leading from DATAFUN clauses to DATALOG clauses. The second step introduces extra arguments for values returned in an is-rhs or in the foot, where new first (not: last) arguments are used to cope with varying-arity DATAFUN ("I"-calls); denotative foots directly become the extra argument of the conclusion while evaluative foots generate a new variable (from $-1,-2, \ldots$ ) used as the extra argument of both the foot and the conclusion. Thus, the relationalized form of the above DATAFUN example is 


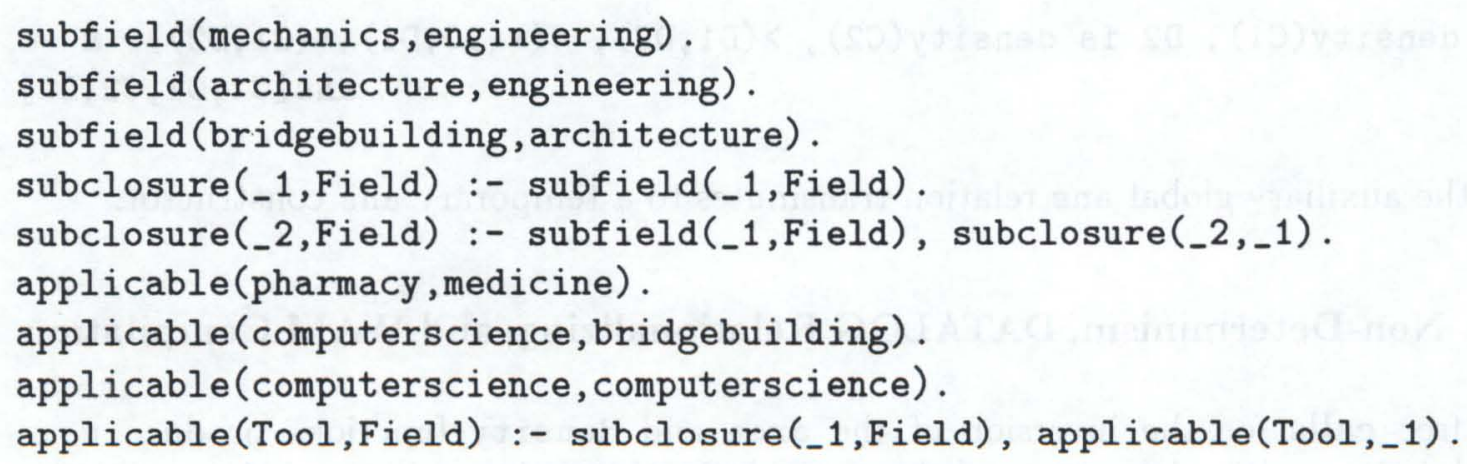

Besides this kind of RELFUN-to-PROLOG translation we have implemented a more direct WAM compilation of non-deterministic, non-ground functions [Bol90]: the WAM temporary register $\mathrm{X} 1$ (identical to the argument register A1) is also used for passing returned values, so that first-argument nestings need not be flattened because the caller directly finds the returned value of the first callee in argument register X1.

\subsection{Full RELFUN Exemplified by "Self"-Functions}

When enriching DATAFUN with structures and lists we arrive at full RELFUN (we will immediately transfer the relational varying-arity extensions). Returning to successor structures for natural numbers, one should first note that it is illegal to nest active calls into passive structures like this: $s[+(M, N)]$. The usual equational definition of binary addition could still be transcribed by employing an is-call for +'s recursion:

$+(0, N):-\& N$.

$+(s[M], N):-A$ is $+(M, N)$ \& $s[A] . \quad($ or $\quad+(s[M], N):-\&+(M, s[N])$.

However, we prefer another method, relying on functions defined to simply return "their own call as a structure". Since the same functor can be a constructor and a defined function, we can define, e.g., s and tup as the following self-passivating functions:

$s(M):-\& \quad s[M]$.

$\operatorname{tup}(\mid Z):-\& \operatorname{tup}[\mid Z]$. (or $\operatorname{tup}(\mid Z):-\&[\mid Z]$. or $\operatorname{tup}(\mid Z):-\& Z$ Z.)

Now, $\mathbf{s}$ and tup may also be called as active functions, evaluating their arguments in the usual call-by-value manner and returning passive structures that use the evaluated arguments as their arguments and the respective function names as their constructors.

For example, the call tup(subfield(engineering), s(s(0))) non-deterministically returns the lists [mechanics, $\mathbf{s}[\mathbf{s}[0]]]$ or [architecture, s[s[0]]]; the LISP-cons-like tup- "I"-use tup $(\mathbf{s}(0) \mid[0])$ returns $[\mathbf{s}[0], 0]$; the COMMON LISP-list*-like tup- "I"use $\operatorname{tup}(\mathrm{a}, \mathrm{b}, \mathrm{c|}[\mathrm{d} e])$ returns $[\mathrm{a}, \mathrm{b}, \mathrm{c}, \mathrm{d}, \mathrm{e}]$. Moreover, the $\mathbf{s}$ definition enables a direct analogue to equational addition:

$+(0, N):-\& N$.

$+(s[M], N):-\& s(+(M, N))$. 
It should also be noted here that RELFUN definitions obey the "constructor discipline" [O'D85], which with our notation amounts to saying simply that "clause heads must not have embedded parenthesized expressions". This would be violated by the eq-nested signum calls shown in the introduction.

The earlier relation-to-function transcriptions (e.g., for the subfield operator) decreased the arity by one because one relation argument was distinguished as the function value. Alternatively, relations can often be refined to functions of the same arity returning an additional useful value. One class of functions generated in this way is filter functions i.e., functions acting as the identity for certain arguments or argument combinations, and failing for other ones. For instance, the sorted relation on lists of subsection 2.2 can be refined to the following filter function, whose recursive call is nested after the "I" into a cons-like tup call:

sorted $([]):-\&[]$.

$\operatorname{sorted}([\mathrm{X}]):-\&[\mathrm{X}]$.

$\operatorname{sorted}([X, Y \mid Z]):-\operatorname{lesseq}(X, Y) \& \operatorname{tup}(X \mid \operatorname{sorted}([Y \mid Z]))$.

This sorted function returns sorted (possibly non-ground) lists like [s[0],E, s[s[0]]] unchanged (up to variable names), and fails for unsorted ones like $[\mathbf{s}[\mathbf{s}[0]], \mathbf{E}, \mathbf{s}[0]]$.

Below, a sample sorted call is given, which occurs in an (internally non-ground and non-deterministic) functional version of the well-known relational slow-sort program [Llo87]. This sort definition also exemplifies an essential use of non-ground function calls: since such calls both bind request variables and return a value, they can be used to split results into bindings, for the calls occurring somewhere above or after them, and a value, for the caller nested directly above them.

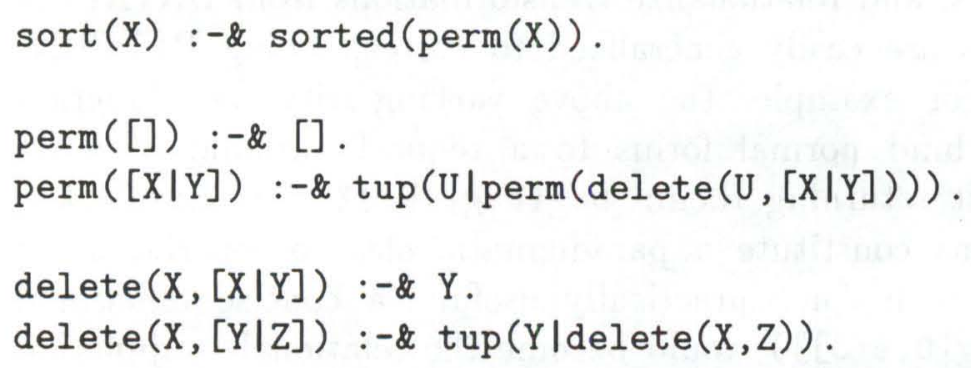

Let us consider this bottom-up. The auxiliary function delete non-deterministically removes occurrences of its first argument from the list in its second argument. The permutation function can then use a non-ground delete call for result splitting: it nondeterministically binds $U$ to arbitrary list elements, for the cons-like tup call, and returns U-less lists, for the recursive perm call. Finally, the sort main function calls the above sorted filter on the non-deterministic permutations of its argument. Note that this functional sort version specifies a computationally preferable (nesting) sequence by calling perm before sorted. In the relational sort specification commutativity of conjunction appears to permit calling sorted before perm, which, however, would not run in normal PROLOGs, as discussed in [Llo87]. A related benefit of the functional formulation is that the computationally less meaningful sort use for 'unsorting' a given sorted list is 
syntactically marked by an is-call over a free sort call, whereas the relational version employs symmetrically-looking non-ground sort calls for both use modes, that would suggest "equality of rights".

A variant of filters is self-testing functions, which can also be viewed as self-passivating functions that yield unknown for an argument (sequence) considered "ill-formed". For example, the varying-arity sorted relation of subsection 2.4 can be refined to a selftesting function that fails for unsorted argument sequences:

sorted(): : \& sorted [].

sorted $(X):-\&$ sorted $[X]$.

$\operatorname{sorted}(X, Y \mid Z)$ :- $\operatorname{lesseq}(X, Y)$, $\operatorname{sorted}[\mid W]$ is $\operatorname{sorted}(Y \mid Z)$ \& $\operatorname{sorted}[X \mid W]$.

Now, the non-ground call sorted $(\mathrm{s}(0), \mathrm{E}, \mathrm{s}(\mathrm{s}[0]))$ returns a renaming variant of sorted $[s[0], E, s[s[0]]]$, while $\operatorname{sorted}(s(s[0]), E, s(0))$ yields unknown.

Concluding the series of "self"-functions, let us proceed to self-normalizing functions, a variant of self-testing functions performing argument normalization. For instance, the previous list sort function can be used to define bag as a varying-arity function that returns a bag structure of the sorted arguments i.e., a normalized multiset:

$\operatorname{bag}(\mid X):-W$ is $\operatorname{sort}(X) \& \operatorname{bag}[\mid W]$.

Now, the call bag $(\mathbf{s}[\mathbf{s}[0]], 0, \mathrm{~s}(\mathrm{~s}[0]), \mathrm{s}(0))$ returns bag $[0, \mathrm{~s}[0], \mathrm{s}[\mathrm{s}[0]], \mathrm{s}[\mathrm{s}[0]]]$. Recalling the discussion in subsection 2.2 , it should be clear that even for a defined function (e.g., bag) no evaluation (e.g., normalization) will happen if it is applied with square brackets: $\operatorname{tup}(\operatorname{bag}(s[0], 0), \operatorname{bag}[s[0], 0])$ returns $[\operatorname{bag}[0, s[0]], \operatorname{bag}[s[0], 0]]$.

The flattening, extra-arguments, and relationalize transformations from DATAFUN to DATALOG in subsection 3.1 .3 are easily generalized to corresponding RELFUNto-PROLOG transformations. For example, the above varying-arity bag function becomes a relation which must bind normal forms to a request variable (the extra first argument) instead of just returning them: $\operatorname{bag}(\operatorname{bag}[\mid W] \mid X):-\operatorname{sort}(W, X)$. However, self-normalizing functions constitute a paradigmatic class of operators for which a relational reformulation seems not practically useful: a concise functional nesting like $\operatorname{set}(\operatorname{bag}(\mathrm{s}[0], 0), \operatorname{bag}[0, s[0]])$ would become the relational conjunction $\operatorname{bag}(B, s[0], 0)$, $\operatorname{set}(S, B, \operatorname{bag}[0, s[0]])$, treating the active and passive bags completely differently, even though they both evaluate to (equal) structures for the set. Again recalling subsection 3.1.3, the X1-reuse for value returning in the WAM also supports full RELFUN because $\mathrm{X} 1$ can point to structured return values on the heap just as it points to structured variable values.

\subsection{Higher-Order Constructors and Functions}

Our derivation of functional programming extensions now arrives at variables and structures used as constructors or functions, and at their combination with non-ground and non-deterministic calls. 
Constructor variables and structures, introduced in a relational context (subsection $2.5)$, are also useful in a functional setting. For instance, a function genints enumerates the integers in the alternating order $0, \pm 1, \pm 2, \ldots$, returned as the infinitely nondeterministic values 0 or $\mathbf{s}[0]$ or $\mathrm{p}[0]$ or $\mathbf{s}[\mathrm{s}[0]]$ or $\mathrm{p}[\mathrm{p}[0]]$ or ... Its definition employs a constructor variable, Sign, for building up a homogeneous, 'absolute' nesting before binding the structure's "neutral signs" (equal in all levels!) to either the successor or predecessor constructor. Instead of using the constants $\mathrm{s}$ and $\mathrm{p}$, we could also apply as constructors the defined functions $1+$ and $1^{-4}$ or structures like inc [1] and inc [-1].

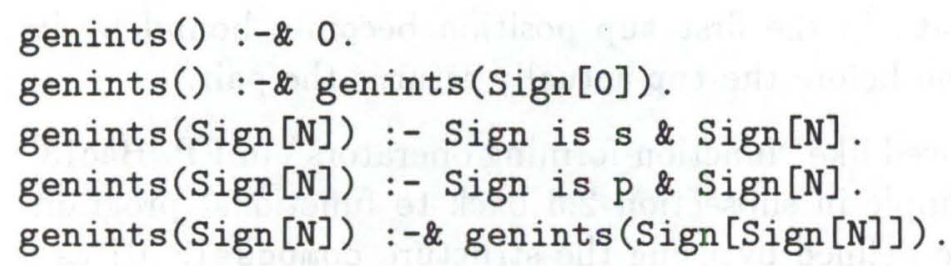

While the main nullary genints/0 generates all integers, the auxiliary unary genints/1 can also be called as genints(Sign [...Sign[0]...]) to generate the integers whose absolute value is not less than the 'absolute' argument, as genints(s[0]) to generate the positive integers, as genints $(\mathrm{p}[0])$ to generate the negative integers, and in other meaningful ways.

Function variables in queries can be utilized much like the corresponding relation variables (see subsection 2.5). For example, given the DATAFUN version of the density database (cf. subsection 3.1.2), the query F (china) asks for all unary properties of china, enumerating the attribute $F=$ area with the returned value 3380 , the attribute $F=$ pop with its value, etc.

Function variables in clauses give us the abstraction power of functional arguments in the fashion of functional programming. Thus, revise is a ternary function applying any unary function $\mathrm{F}$ to the $\mathrm{N}$ th element of a list (for $\mathrm{N}$ greater than the list length or $\mathrm{N}$ less than 1 it returns the list unchanged):

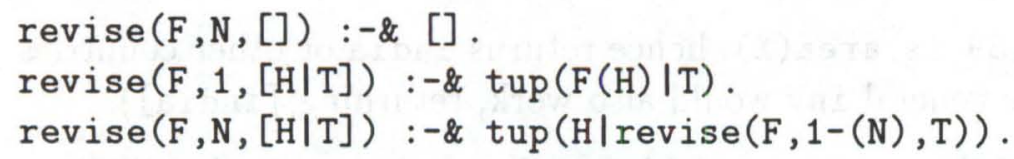

Similarly, the sort function could be parameterized by a Compare relation to be handed to the sorted filter, which would abstract from the specific lesseq relation (in particular, from the representation of naturals as s structures). Of course, this "functional style" of universally quantified operator variables, occurring on both sides of definitions, is also useful in purely relational examples. Conversely, the "relational style" of existentially quantified operator variables, occurring only on the rhs of definitions, would also be useful

\footnotetext{
${ }^{4}$ RELFUN accesses a selected subset of COMMON LISP functions as builtins. Unusually named examples are the numeric successor and predecessor functions $1+$ and 1-, whose application to an argument, say 6 , in mathematical or PROLOG syntax becomes $1+(6)$ and 1-(6), returning 7 and 5, respectively. RELFUN's ecal primitive, a combination of LISP's eval and PROLOG's metacall, permits the activation of structures, as in ecal $(1-[1-[0]])$, returning -2 .
} 
in purely functional examples. Thus, the earlier function-variable query about china could be further abstracted for use in the rhs of a rule returning attribute/value pairs of an object. The below attval function employs both the rhs-only variable Attribute and an lhs/rhs variable Valfilter (bound, e.g., to numfilter) for filtering the values returned by Attribute:

attval (Obj,Valfilter) :-\& tup(Attribute, Valfilter(Attribute(Obj))). numfilter $(X)$ :- numberp $(X)$ \& $X$.

Note that the free variable Attribute in the first tup position becomes bound by its application in the second tup position before the tup actually returns the pair.

Function structures can be employed like "function-forming operators" in FP [Bac78]. Bringing the relational-product example in subsection 2.5 back to functional programming, functional composition can be defined by using the structure compose [F, G] as a function, which permits twice to be defined as a compose-structure-valued footed fact:

compose $[F, G](X):-\& F(G(X))$.

twice $(F):-\&$ compose $[F, F]$.

Again, while the structure compose[...] can be (higher-order-)called directly, as in compose [fathfun, mothfun] ( $j \circ h n$ ), the constant twice is (first-order-)called in function position to return an applicable function structure, as in twice (fathfun) ( $j \circ h n$ ).

Let us now turn to the combination of higher-order operators with non-ground and non-deterministic calls.

For example, $\mathrm{F}^{-1}$, the inversion of a unary function $\mathrm{F}$, can be defined as a function structure inv $[\mathrm{F}]$ which calls $\mathrm{F}$ freely within an is-call only accepting $\mathrm{F}$ values that match the argument $\mathrm{V}$ of $\mathrm{F}^{-1}$ (for an $\mathrm{N}$-ary $\mathrm{F}$ we just add a "I"):

$\operatorname{inv}[F](V):-V$ is $F(X)$ \& $X . \quad$ (generally $\operatorname{inv}[F](V):-V$ is $F(\mid X)$ \& $X$.

Thus, inv[area] (1139) calls 1139 is area(X), hence returns india or other countries for which area returns 1139 (the general inv would also work, returning [india]).

Another example, a version of the $\mu$-operator, additionally employs a result-splittinglike technique (used in the sort definition) to fork the entire result of a call into both the binding of a request variable and the returned value. First, we define a non-deterministic generator naturals, enumerating the naturals from an initialization given in the first argument, where the next natural is always both bound to the second argument and returned.

naturals $(\mathrm{N}, \mathrm{N}):-\& \mathrm{~N}$.

naturals $(N, V):-\&$ naturals $(1+(N), V)$.

For instance, naturals $(3, \mathrm{~V})$ binds $\mathrm{V}$ to 3 or 4 or ...; at the same time it returns each of these values. This then permits to concisely define a non-deterministic mu higher-order 
function taking unary functions over the naturals as its argument and returning their smallest argument for which they return 0, then their second-smallest argument, etc., diverging if there is none $\left(\operatorname{left}^{5}\right)$ :

$\mathrm{mu}(\mathrm{F}):-0$ is $F($ naturals $(0, \mathrm{~V}))$ \& $\mathrm{V}$.

The ap reduction of higher-order relations in subsection 2.5 directly transfers to function variables and structures. For instance, the above rule could be reduced to

$\operatorname{ap}(m u, F):-0$ is $\operatorname{ap}(F, \operatorname{ap}($ naturals $, 0, V))$ \& $V$.

changing $\mathrm{F}$ from a function variable into an argument variable. The effects on the WAM implementation are the same as discussed for higher-order relations.

\section{The Logic/Functional Style in Use}

Recent RELFUN projects have explored the use of relational/functional programming for non-toy problems: the language has been evaluated and tuned by programs for realistic tasks such as hypergraph processing [Bol92] and NC-program generation [ $\left.\mathrm{BHH}^{+} 91\right]$. In order to facilitate comparison with other languages, this section gives versions of three well-known non-trivial programs in RELFUN's logic/functional style (some features of RELFUN, most notably higher-order operations, will not be needed in these examples).

\section{1 serialise: Inplace Updates of Non-Ground Structures}

After the density database, the second practical PROLOG example given in [WPP77] is the relational serialise program. Its task is to transform a list of items into a corresponding list of their alphabetic serial numbers; e.g., $[p, r, 0,1,0, g]$ should become $[4,5,3,2,3,1]$.

The subrelations of serialise demonstrate the use of the "logical" variable: first pairlists binds a request variable to a non-ground list of free variables (the prospective answer list), e.g. [Y1 , Y2 , Y3, Y4 , Y5 , Y6], and another request variable to a corresponding list of non-ground structures, e.g. [pair $[\mathrm{p}, \mathrm{Y} 1]$, pair $[r, Y 2], \operatorname{pair}[0, Y 3], \operatorname{pair}[1, Y 4]$, pair $[0, Y 5]$, pair $[g, Y 6]]$, thus generating two variable-coupled "incomplete data structures"; then arrange (quick)sorts the list of pairs into a binary tree, calling a partition relation that uses the items in the first pair arguments for (string) comparison; now

\footnotetext{
${ }^{5}$ RELFUN's once primitive (or "!" symbol) could be employed above (or after) the is-call to prevent the possibly diverging search for further solutions when the first solution is found, thus simulating the usual - unbounded - minimalization of unary functions [for $(1+\mathrm{N})$-ary functions "I" can be used to minimalize over the first argument, making $m u[F]$ a function structure, as shown in square brackets]:

$\operatorname{mu}(F):-\operatorname{once}(0$ is $F(n a t u r a l s(0, V))$ ) V. (or $\operatorname{mu}(F):-0$ is $F($ naturals $(0, V))$ ! V.)

[mu[F] (|Nargs) :- $\operatorname{once}(0$ is $F(n a t u r a l s(0, V) \mid \operatorname{Nargs}))$ V.]
} 
numbered can count the items, left to right, at the fringe of the tree and note the resulting serial numbers by "inplace updates" in the second pair arguments, which by logicalvariable equality instantiate pairlists' prospective answer list to the final, ground result.

Although this binary serialise relation can be called like serialise ( $[\mathrm{p}, \mathrm{r}, 0,1,0, \mathrm{~g}]$, $[4,5,3,2,3,1])$, to check the relationship, and like serialise $([p, r, 0,1,0, g], S)$, to generate the list of serial numbers, it cannot be called like serialise (,$[4,5,3,2,3,1]$ ), to generate all item lists mapping to given serial numbers (the comparison relation expects constant items): the main serialise algorithm just employs a relational syntax to express a non-invertible function from item lists to serial-number lists, while it does make an essential, "two-results" use of the subrelation partition and of intermediate non-ground terms.

Therefore it appears natural to reformulate serialise in RELFUN's non-ground functional style, keeping the partition relation and intermediate non-ground returned values ${ }^{6}$. Relationship-checking calls will then look like $[4,5,3,2,3,1]$ is serialise $([p, r, o, l, o, g])$, an is-call containing a ground function call for serialnumber generation as its rhs. The above explanation for the relational version can be transferred to this functional one by noting that the "principal result" is now always returned as a value instead of being bound to a request variable: the pairlists non-ground function only binds its prospective-answer result for use as serialise's foot premise $R$, but directly returns the list of pairs to the arrange function, which again returns its non-ground tree to the first argument of the self-nested numbered function.

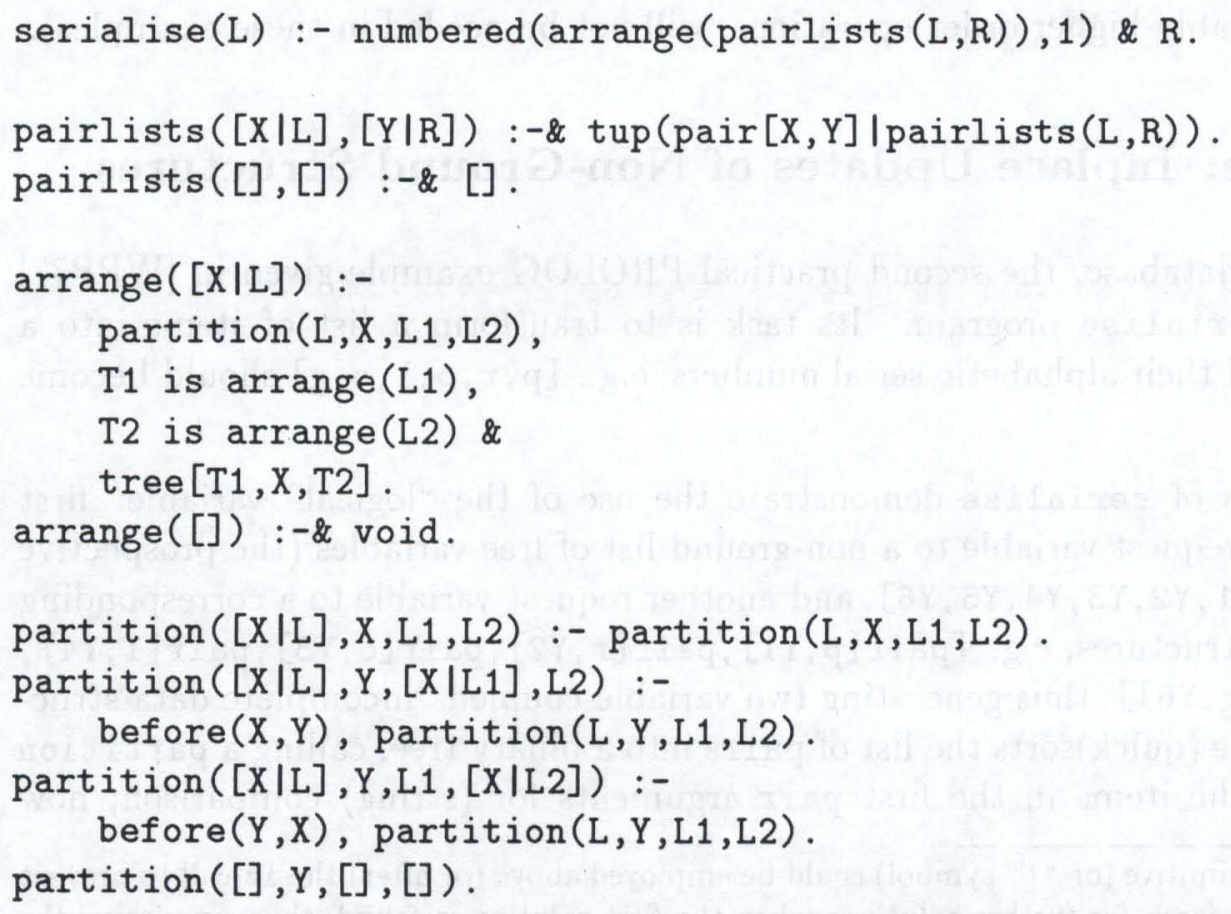

\footnotetext{
${ }^{6}$ While the need for non-ground terms is self-evident in relational programming (ground relational programming isn't very useful), they require some justification in functional programming. The serialise example shows how non-ground terms can be useful internally in a computation even if its external input/output is ground terms. This is analogous to an internal use of complex numbers in computing real results.
} 


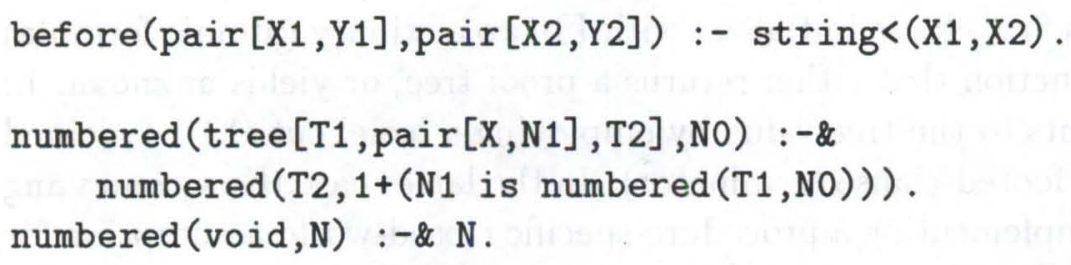

Note that the body of the first arrange clause can be simplified to partition (L , X, L1, L2) \& tree (arrange(L1), $\mathrm{X}$, arrange(L2)) if tree is defined as a self-passivating function or, similarly, if 3-tups are used instead of labeled binary trees (cf. subsection 3.2). Also notice that numbered 'updates' the pair structures at the roots of the tree structures by is-binding the unavoidable logical variable $\mathrm{N} 1$ to the recursion result obtained from traversing the left subtree $\mathrm{T} 1$, a value which is incremented by $1+$ for use in traversing the right subtree T2. This works since RELFUN's is-builtin both binds and returns the value of its rhs.

\section{2 wang: On-the-Fly Construction of Proof Trees}

Since its pure LISP description in [MAE ${ }^{+} 62$ ], Wang's proof algorithm for the propositional calculus has often been reformulated to demonstrate the use of declarative languages. The algorithm applies reduction rules to a sequent representation of propositional formulas until an atomic formula occurs in both the antecedent and consequent of all derived sequents, reporting true, or no more rule is applicable to a sequent, reporting false. [PS91] gives a version with an extra relation argument for constructing a proof tree "onthe-fly", whose size can be computed by an invertible function.

Here we give a RELFUN version that returns the trees of successful proofs, where subtrees are built and their roots labeled "on-the-fly" by a constructor and two selfpassivating functions: the constructor indicates that an atomic formula occurs on both sequent sides and the self-passivating functions exhibit the reduction of a formula on the right (consequent) or on the left (antecedent) side.

For example, $\operatorname{wang}([],[\operatorname{impl}[\operatorname{and}[p$, and $[q, r]]$, and $[$ and $[p, q], r]]]$ ) returns the and-associativity proof tree

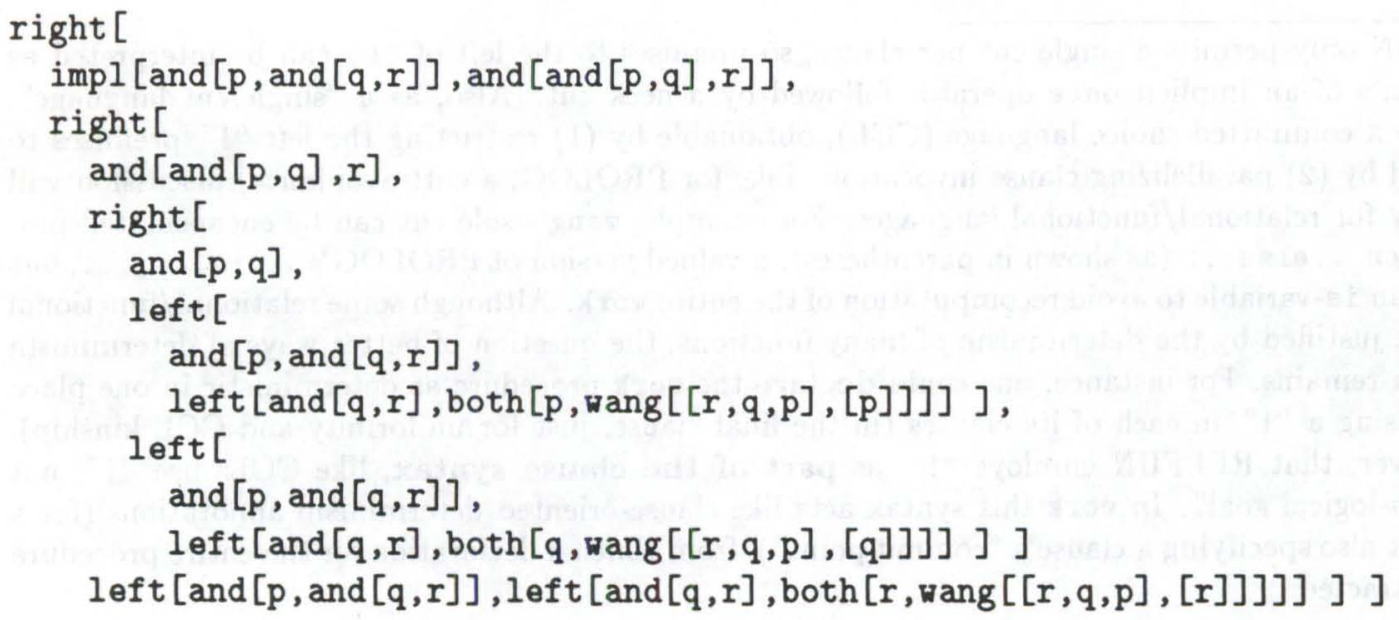




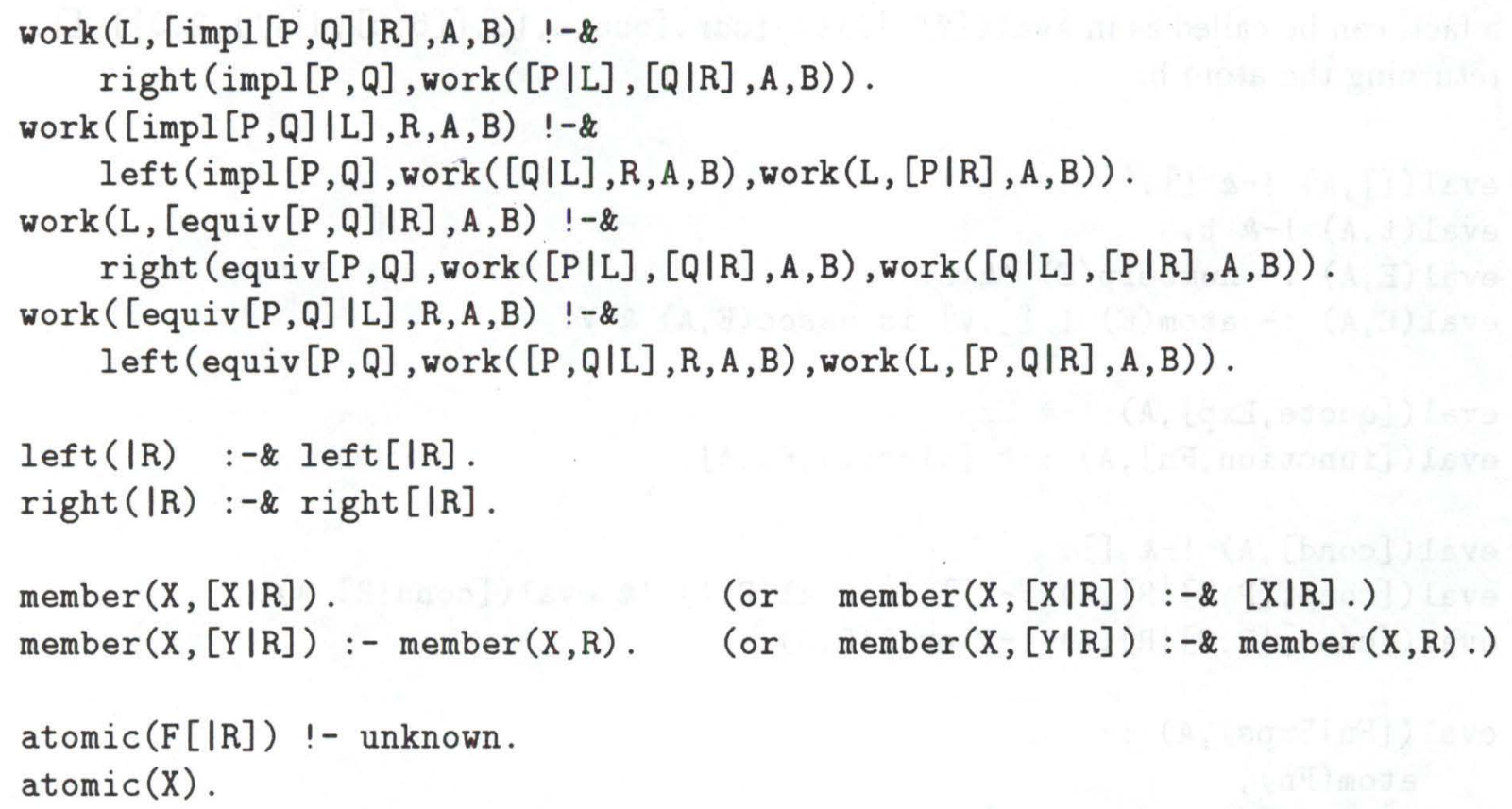

The alternative member definition (using both parenthesized clauses) is the LISP-like version mentioned in the introduction. (Using the first parenthesized clause and the second unparenthesized clause gives a definition returning $[\mathrm{X} \mid \mathrm{R}]$ instead of true only for an $\mathrm{X}$ occurring as the first element of the original list.) Our functional wang algorithm could again be degenerated to a non-tree-building relational algorithm by just omitting all "\&"-separators; the resulting hornish clauses could then be simplified, mainly by bringing the work recursions to the top-level.

\section{3 eval: Interpreting a LISP Subset in RELFUN}

Most LISP-in-LISP metainterpreters descended from the metacircular eval/apply specification of LISP 1.5 [MAE $\left.{ }^{+} 62\right]$. The operational semantics of pure LISP was later transcribed to a concise pure PROLOG relation eval [PP82]. The below deterministic RELFUN function eval, corecursive with apply, defines, without concern for efficiency, a non-trivial LISP subset including closures, macros, and an object-level eval ${ }^{8}$.

LISP lists (and function calls) are represented as RELFUN lists (with distinguished first elements). As usual, lists with first element lambda are interpreted as temporary functions. Permanent functions (and macros) become relational defun (and defmacro) facts from which calls extract lambda functions.

For instance, defun (ff, $[\mathrm{x}],[$ cond $,[[\operatorname{atom}, \mathrm{x}], \mathrm{x}],[\mathrm{t},[\mathrm{ff},[\mathrm{car}, \mathrm{x}]]]])$, asserted as

\footnotetext{
${ }^{8}$ We do not try here to capture the LISP subset in RELFUN which is required for our implementation of RELFUN in LISP; it would need some profane features for reading/printing etc., but could avoid the advanced features mentioned. This would provide a 'codefinition' of RELFUN and LISP, like the one proposed for PROLOG and LISP in Kenneth M. Kahn's "Pure Prolog in Pure Lisp" response (Logic Programming Newsletter 5, Winter 83/84) to the "Pure Lisp in Pure Prolog" [PP82] paper. A direct definition of RELFUN in RELFUN has been prepared by reducing it to a meaning-preserving kernel (via flattening or relationalizing), for which a PROLOG-like (vanilla) metainterpreter can be given.
} 
a fact, can be called as in eval ( $[\mathrm{ff},[$ ist, $[\mathrm{cdr},[$ quote,$[a,[[b, c], d]]]], 2,3]],[])$, returning the atom $b$.

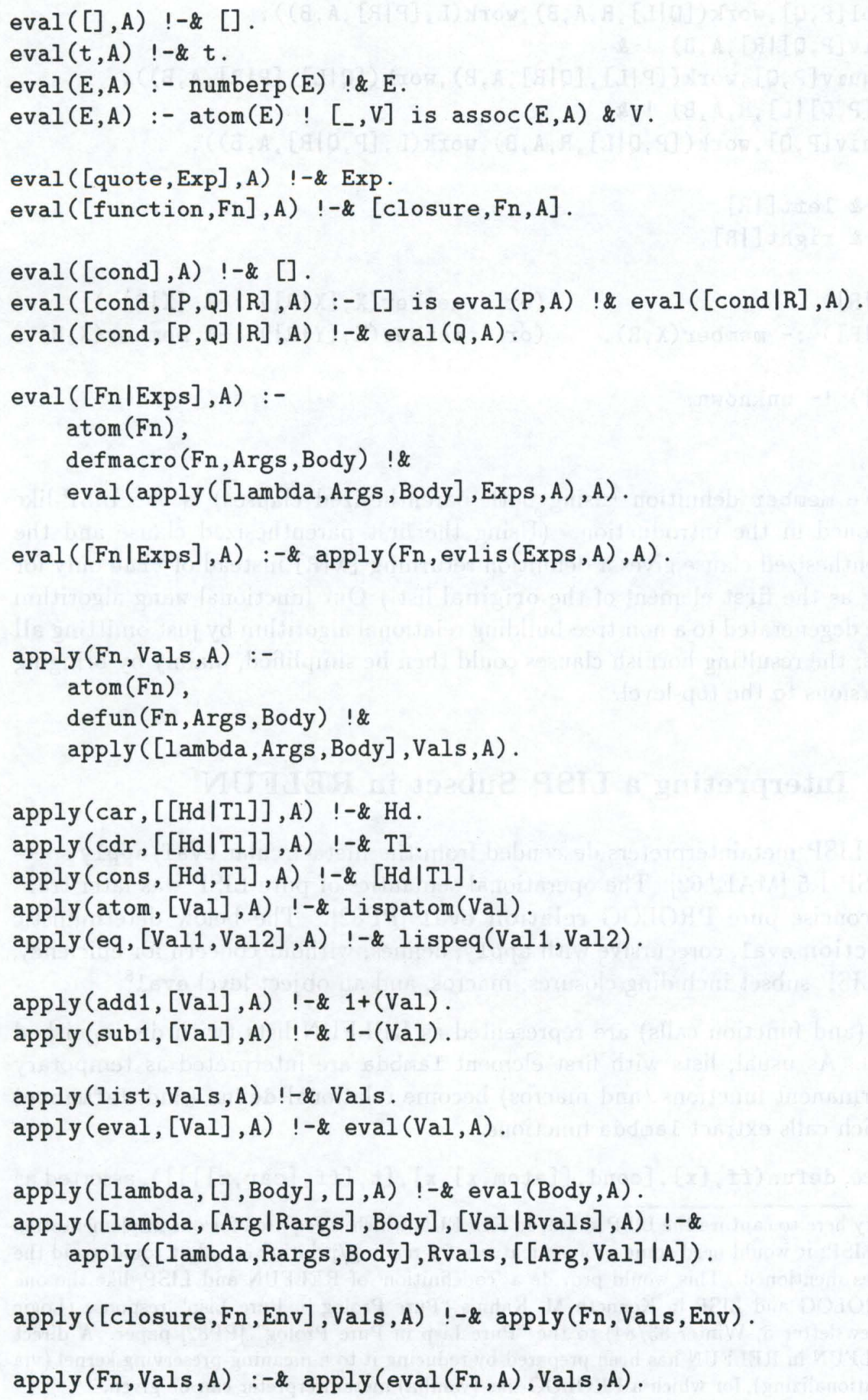




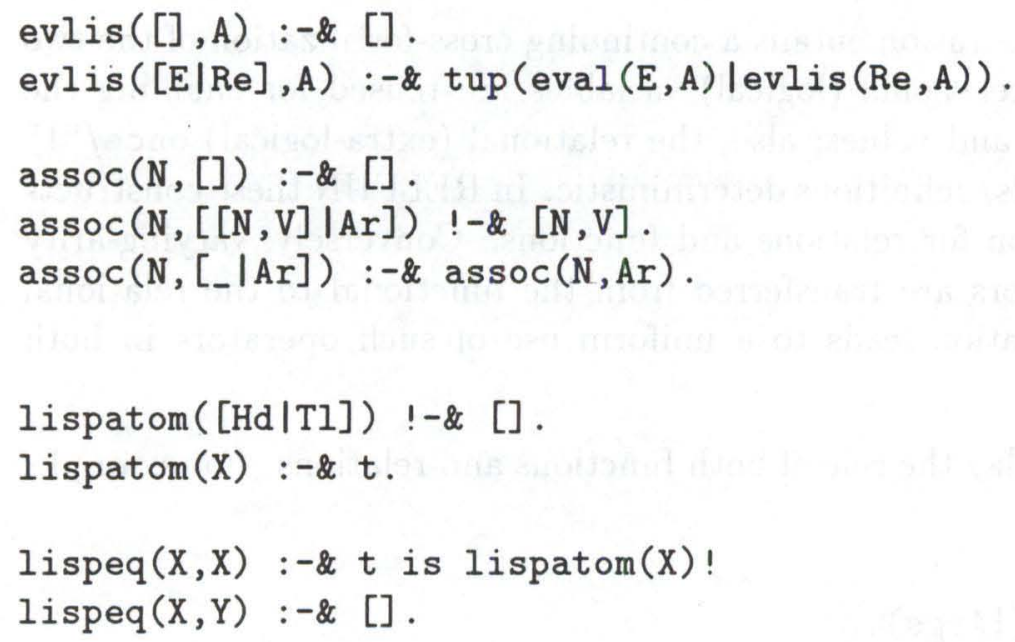

This LISP interpreter performs more well-formedness checks than most LISP-based ones: the correct number and structure of arguments is verified by unification (e.g., quote should have exactly one argument), yielding unknown for ill-formed expressions. The eval function corecurses with the usual evlis auxiliary to evaluate actual arguments; for uniformity, even arguments of special forms and macros are submitted to evlis (in the final eval clause) iff their function is itself the result of an evaluation (in the final apply clause). The specification has no need for the usual pairlis auxiliary because a lambda application leads to an apply recursion through the lambda-argument and actualvalue lists; that the Arg/Val pairs thus extend the environment, $\mathrm{A}$, in reversed order does not matter for legal LISP operators, having no duplicate lambda variables (as usual, our interpreter does not prevent formal-argument repetitions; however, by reversing the pair order in the A-list it effects LISP's normal left-to-right evaluation even on lambda binding).

\section{Conclusions}

The RELFUN research attempts to combine and extend programming concepts and techniques that have accumulated in the relational (principally, PROLOG) and functional (prototypically, LISP) communities.

A comprehensive subset of PROLOG is kept as a sublanguage with little syntactic modification (structures written with square brackets instead of parentheses, cut used as a separator instead of a goal). This basis is then systematically extended by advanced relational notions, a rich set of functional notions, and a combination of both.

The functional sublanguage of RELFUN is much influenced by the implementation language LISP. But as in newer functional languages, like ML and MIRANDA, a function is defined by "pattern $\rightarrow$ action" clauses instead of a conditional expression. Generalizing pattern matching to unification, RELFUN permits non-ground functions, as allowed in other logic/functional integrations [DL86]. This also leads to non-deterministic functions, enumerating finitly or infinitly many values via backtracking. 
The relational/functional integration entails a continuing cross-fertilization of the two language styles. For instance, relational (logical) variables are reused for enabling the non-ground function arguments and values; also, the relational (extra-logical) once/"!" is reused for making function calls/definitions deterministic. In RELFUN these constructs are employed in the same fashion for relations and functions. Conversely, varying-arity and certain higher-order operators are transferred from the functional to the relational world. Again, the cross-fertilization leads to a uniform use of such operators in both sublanguages.

In fact, some operators can play the role of both functions and relations. For example, the concise pair of clauses

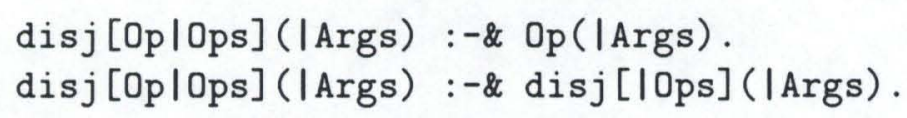

defines disj as a varying-arity, higher-order, non-ground, non-deterministic function structure that recursively applies its operator parameters op $1, \ldots$ (one or more relations or functions) to zero or more (possibly non-ground) arguments arg $1, \ldots$, enumerating the (possibly non-ground) values of op $1(\arg 1, \ldots), \ldots$ A dis $j$ call fails if none of these operator calls successfully returns a value, hence we have at the same time defined a disjunction relation of 'success'/'failure' logic. (A cut ending the first clause would prevent functional value enumeration as well as relational truth multiplicity after the first success.)

Summarizing, RELFUN provides a tunable system of relational/functional language extensions, which can be used in isolation and in free combination. In particular, this holds for the orthogonal functional, varying-arity, higher-order, and cut extensions of the pure-PROLOG-like kernel. Several other extensions of pure PROLOG, e.g. types (incl. finite domains) and modules, being quite independent from the ones in RELFUN, could probably be added without difficulty, e.g. leading to typed values as well as arguments.

Besides the 'dynamic' interplay between our language extensions, there are 'static' reduction possibilities for several of them. Most notably, the functional sublanguage can be relationalized and the higher-order part can be reduced to the first-order part. While with these reductions RELFUN's semantics is indirectly founded on the usual Herbrand models for Horn clauses, the author is working on a more direct characterization of RELFUN's first-order hornish and footed clauses using functionally extended Herbrand models (instead of distinguishing an equality relation). The 'horizontal' transformations of the full language into a kernel are also important in preparation for RELFUN's 'vertical' WAM compilation. While the complete language is implemented as an interpreter, a firstorder subset is realized as a compiler/emulator. The RELFUN sources are available in (a portable subset of) COMMON LISP along with the program samples of this paper.

In our hybrid expert-system shell, COLAB, RELFUN's backward rules are augmented by forward rules, taxonomies, and constraints [BHHM91]. Problems of realistic size are now being solved by RELFUN [Bol92] and RELFUN/COLAB [BHH $\left.{ }^{+} 91\right]$ programs. 


\section{References}

[Bac78] John Backus. Can programming be liberated from the von Neumann style? A functional style and its algebra of programs. CACM, 21(8):613-641, August 1978.

[BGM88] P.G. Bosco, E. Giovannetti, and C. Moiso. Narrowing vs. SLD-resolution. Theoretical Computer Science, 59:3-23, 1988.

[BHH ${ }^{+}$11] Harold Boley, Philipp Hanschke, Martin Harm, Knut Hinkelmann, Thomas Labisch, Manfred Meyer, Joerg Mueller, Thomas Oltzen, Michael Sintek, Werner Stein, and Frank Steinle. $\mu$ CAD2NC: A declarative latheworkplanning model transforming CAD-like geometries into abstract NC programs. Technical Report Document D-91-15, University of Kaiserslautern, DFKI, November 1991.

[BHHM91] H. Boley, P. Hanschke, K. Hinkelmann, and M. Meyer. COLAB: A Hybrid Knowledge Compilation Laboratory. 3rd International Workshop on Data, Expert Knowledge and Decisions: Using Knowledge to Transform Data into Information for Decision Support, September 1991.

[Bol86] Harold Boley. RELFUN: A relational/functional integration with valued clauses. SIGPLAN Notices, 21(12):87-98, December 1986.

[Bol90] Harold Boley. A relational/functional language and its compilation into the WAM. Technical Report SEKI SR-90-05, University of Kaiserslautern, Department of Computer Science, April 1990.

[Bol92] Harold Boley. Declarative operations on nets. In Fritz Lehmann, editor, Semantic Networks in Artificial Intelligence. Special Issue of Computers \& Mathematics with Applications, Pergamon Press, 1992. Preprinted as: DFKI Research Report RR-90-12, Oct. 1990.

[CK85] Mats Carlsson and Kenneth M. Kahn. LM-Prolog user manual. Technical Report UPMAIL 24, Uppsala University, Department of Computer Science, Revised April 1985.

[DL86] D. DeGroot and G. Lindstrom, editors. Logic Programming: Functions, Relations, and Equations. Prentice-Hall, 1986.

[Fri84] Laurent Fribourg. Oriented equational clauses as a programming language. $J$. Logic Programming, 1(2):165-177, 1984.

[Fri85] Laurent Fribourg. SLOG: A logic programming language interpreter based on clausal superposition and rewriting. In 1985 Symposium on Logic Programming, pages 172-184. IEEE Computer Society Press, 1985. 
[GF91] Michael R. Genesereth and Richard Fikes in collaboration with Danny Bobrow, Piero Bonissone, Ron Brachman, Ramanathan Guha, Reed Letsinger, Valdimir Lifschitz, Bob MacGregor, John McCarthy, Peter Norvig, Ramesh Patil, and Len Schubert. Knowledge Interchange Format Version 2.2 Reference Manual. Technical Report Logic-90-4, Stanford University, Computer Science Department, Logic Group, March 1991.

[GM84] Joseph A. Goguen and José Meseguer. Equality, types, modules, and (why not?) generics for logic programming. J. Logic Programming, 1(2):179-210, 1984 .

[Llo87] John W. Lloyd. Foundations of Logic Programming. Springer-Verlag, Berlin, Heidelberg, New York, 1987.

[MAE $\left.{ }^{+} 62\right]$ John McCarthy, Paul W. Abrahams, Daniel J. Edwards, Timothy P. Hart, and Michael I. Levin. LISP 1.5 programmer's manual. MIT Press, Cambridge, Mass., 1962.

[NM90] Gopalan Nadathur and Dale Miller. Higher-order Horn clauses. JACM, 37(4):777-814, October 1990.

[O'D85] M. J. O'Donnell. Equational Logic as a Programming Language. MIT Press, Cambridge, Mass., 1985.

[PP82] Luís Moniz Pereira and António Porto. Pure Lisp in pure Prolog. Logic Programming Newsletter 3, Summer 1982. Universidade Nova de Lisboa, Departamento de Informática.

[PS91] Lawrence C. Paulson and Andrew W. Smith. Logic programming, functional programming, and inductive definitions. In P. Schroeder-Heister, editor, Extensions of Logic Programming, pages 283-309, Berlin, Heidelberg, New York, 1991. Springer-Verlag. LNCS 475.

[Sin92] Michael Sintek. Generalized indexing methods for higher-order PROLOG extensions: A case study with the RELFUN WAM. Technical report, University of Kaiserslautern, DFKI, Forthcoming 1992.

[War82] David H. D. Warren. Higher-order extensions to PROLOG: Are they needed? Machine Intelligence, 10:441-454, 1982.

[WPP77] David H. D. Warren, Luis M. Pereira, and Fernando Pereira. Prolog - the language and its implementation compared with Lisp. SIGPLAN Notices, 12(8):109-115, August 1977. Special Issue. 


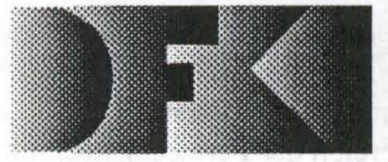

Deutsches

Forschungszentrum

für Künstliche

Intelligenz GmbH

\section{DFKI Publikationen}

Die folgenden DFKI Veröffentlichungen sowie die aktuelle Liste von allen bisher erschienenen Publikationen können von der oben angegebenen Adresse bezogen werden.

Die Berichte werden, wenn nicht anders gekennzeichnet, kostenlos abgegeben.

\section{DFKI Research Reports}

\section{RR-90-15}

Harald Trost: The Application of Two-level Morphology to Non-concatenative German Morphology

13 pages

\section{RR-90-16}

Franz Baader, Werner Nutt: Adding Homomorphisms to Commutative/Monoidal Theories, or: How Algebra Can Help in Equational Unification 25 pages

\section{R R -90 - 17}

Stephan Busemann:

Generalisierte Phasenstrukturgrammatiken und ihre Verwendung zur maschinellen Sprachverarbeitung 114 Seiten

\section{RR-91-01}

Franz Baader, Hans-Jürgen Bürckert, Bernhard Nebel, Werner Nutt, Gert Smolka: On the Expressivity of Feature Logics with Negation, Functional Uncertainty, and Sort Equations 20 pages

\section{RR-91-02}

Francesco Donini, Bernhard Hollunder, Maurizio Lenzerini, Alberto Marchetti Spaccamela, Daniele Nardi, Werner Nutt: The Complexity of Existential Quantification in Concept Languages

22 pages

\section{RR-91-03}

B.Hollunder, Franz Baader: Qualifying Number

Restrictions in Concept Languages

34 pages

RR-91-04

Harald Trost: X2MORF: A Morphological Component Based on Augmented Two-Level Morphology

19 pages
DFKI

-Bibliothek-

PF 2080

D-6750 Kaiserslautern

FRG

\section{DFKI Publications}

The following DFKI publications or the list of all publisched papers so far can be ordered from the above address.

The reports are distributed free of charge except if otherwise indicated.

\section{RR-91-05}

Wolfgang Wahlster, Elisabeth André, Winfried

Graf, Thomas Rist: Designing Illustrated Texts: How Language Production is Influenced by Graphics Generation.

17 pages

\section{RR-91-06}

Elisabeth André, Thomas Rist: Synthesizing Illustrated Documents: A Plan-Based Approach 11 pages

\section{RR-91-07}

Günter Neumann, Wolfgang Finkler: A HeadDriven Approach to Incremental and Parallel Generation of Syntactic Structures 13 pages

\section{RR-91-08}

Wolfgang Wahlster, Elisabeth André, Som Bandyopadhyay, Winfried Graf, Thomas Rist: WIP: The Coordinated Generation of Multimodal Presentations from a Common Representation 23 pages

RR-91-09

Hans-Jürgen Bürckert, Jürgen Müller, Achim Schupeta: RATMAN and its Relation to Other Multi-Agent Testbeds

31 pages

\section{RR-91-10}

Franz Baader, Philipp Hanschke: A Scheme for Integrating Concrete Domains into Concept Languages

31 pages

\section{RR-91-11}

Bernhard Nebel: Belief Revision and Default Reasoning: Syntax-Based Approaches 37 pages 
RR-91-12

J.Mark Gawron, John Nerbonne, Stanley Peters:

The Absorption Principle and E-Type Anaphora

33 pages

\section{RR-91-13}

Gert Smolka: Residuation and Guarded Rules for Constraint Logic Programming

17 pages

RR-91-14

Peter Breuer, Jürgen Müller: A Two Level

Representation for Spatial Relations, Part I

27 pages

RR-91-15

Bernhard Nebel, Gert Smolka:

Attributive Description Formalisms ... and the Rest of the World

20 pages

\section{RR-91-16}

Stephan Busemann: Using Pattern-Action Rules for the Generation of GPSG Structures from Separate Semantic Representations

18 pages

\section{R R-91-17}

Andreas Dengel, Nelson M. Mattos:

The Use of Abstraction Concepts for Representing and Structuring Documents

17 pages

RR-91-18

John Nerbonne, Klaus Netter, Abdel Kader Diagne, Ludwig Dickmann, Judith Klein:

A Diagnostic Tool for German Syntax

20 pages

RR-91-19

Munindar P. Singh: On the Commitments and Precommitments of Limited Agents

15 pages

RR-91-20

Christoph Klauck, Ansgar Bernardi, Ralf Legleitner FEAT-Rep: Representing Features in CAD/CAM 48 pages

RR-91-21

Klaus Netter: Clause Union and Verb Raising Phenomena in German

38 pages

RR-91-22

Andreas Dengel: Self-Adapting Structuring and Representation of Space

27 pages
RR-91-23

Michael Richter, Ansgar Bernardi, Christoph

Klauck, Ralf Legleitner: Akquisition und Repräsentation von technischem Wissen für Planungsaufgaben im Bereich der Fertigungstechnik 24 Seiten

RR-91-24

Jochen Heinsohn: A Hybrid Approach for Modeling Uncertainty in Terminological Logics 22 pages

\section{R R-91-25}

Karin Harbusch, Wolfgang Finkler, Anne Schauder: Incremental Syntax Generation with Tree Adjoining Grammars

16 pages

RR-91-26

M. Bauer, S. Biundo, D. Dengler, M. Hecking, J. Koehler, G. Merziger:

Integrated Plan Generation and Recognition - A Logic-Based Approach -

17 pages

R R -91-27

A. Bernardi, H. Boley, Ph. Hanschke,

K. Hinkelmann, Ch. Klauck, O. Kühn,

R. Legleitner, M. Meyer, M. M. Richter,

F. Schmalhofer, G. Schmidt, W. Sommer:

ARC-TEC: Acquisition, Representation and Compilation of Technical Knowledge 18 pages

RR-91-28

Rolf Backofen, Harald Trost, Hans Uszkoreit:

Linking Typed Feature Formalisms and Terminological Knowledge Representation Languages in Natural Language Front-Ends 11 pages

RR-91-29

Hans Uszkoreit: Strategies for Adding Control Information to Declarative Grammars 17 pages

RR-91-30

Dan Flickinger, John Nerbonne:

Inheritance and Complementation: A Case Study of Easy Adjectives and Related Nouns 39 pages

RR-91-31

H.-U. Krieger, J. Nerbonne:

Feature-Based Inheritance Networks for Computational Lexicons

11 pages

RR -91-32

Rolf Backofen, Lutz Euler, Günther Görz: Towards the Integration of Functions, Relations and Types in an AI Programming Language 14 pages 
RR-91-33

Franz Baader, Klaus Schulz:

Unification in the Union of Disjoint Equational

Theories: Combining Decision Procedures

33 pages

\section{R R-91-34}

Bernhard Nebel, Christer Bäckström:

On the Computational Complexity of Temporal

Projection and some related Problems

35 pages

\section{RR-91-35}

Winfried Graf, Wolfgang Maaß: Constraint-basierte Verarbeitung graphischen Wissens

14 Seiten

\section{RR-92-03}

Harold Boley:

Extended Logic-plus-Functional Programming 28 pages

\section{R R-92-04}

John Nerbonne: Feature-Based Lexicons:

An Example and a Comparison to DATR

15 pages

\section{RR-92-05}

Ansgar Bernardi, Christoph Klauck,

Ralf Le gleitner, Michael Schulte, Rainer Stark:

Feature based Integration of CAD and CAPP

19 pages

\section{DFKI Technical Memos}

TM-91-01

Jana Köhler: Approaches to the Reuse of Plan

Schemata in Planning Formalisms

52 pages

TM-91-02

Knut Hinkelmann: Bidirectional Reasoning of Horn Clause Programs: Transformation and Compilation 20 pages

\section{TM-91-03}

Otto Kühn, Marc Linster, Gabriele Schmidt:

Clamping, COKAM, KADS, and OMOS:

The Construction and Operationalization

of a KADS Conceptual Model

20 pages

\section{TM-91-04}

Harold Boley (Ed.):

A sampler of Relational/Functional Definitions 12 pages
TM-91-05

Jay C. Weber, Andreas Dengel, Rainer Bleisinger: Theoretical Consideration of Goal Recognition Aspects for Understanding Information in Business Letters

10 pages

\section{TM-91-06}

Johannes Stein: Aspects of Cooperating Agents 22 pages

\section{TM-91-08}

Munindar P. Singh: Social and Psychological

Commitments in Multiagent Systems

11 pages

TM-91-09

Munindar P. Singh: On the Semantics of Protocols Among Distributed Intelligent Agents 18 pages

TM-91-10

Béla Buschauer, Peter Poller, Anne Schauder, Karin Harbusch: Tree Adjoining Grammars mit

Unifikation

149 pages

\section{TM-91-11}

Peter Wazinski: Generating Spatial Descriptions for Cross-modal References

21 pages

TM-91-12

Klaus Becker, Christoph Klauck, Johannes Schwagereit: FEAT-PATR: Eine Erweiterung des D-PATR zur Feature-Erkennung in CAD/CAM 33 Seiten

\section{TM-91-13}

Knut Hinkelmann:

Forward Logic Evaluation: Developing a Compiler from a Partially Evaluated Meta Interpreter 16 pages

\section{TM-91-14}

Rainer Bleisinger, Rainer Hoch, Andreas Dengel: ODA-based modeling for document analysis 14 pages

\section{TM-91-15}

Stefan Bussmann: Prototypical Concept Formation An Alternative Approach to Knowledge Representation

28 pages

TM-92-01

Lijuan Zhang:

Entwurf und Implementierung eines Compilers zur Transformation von Werkstückrepräsentationen 34 Seiten 


\section{DFKI Documents}

\section{D-91-01}

Werner Stein, Michael Sintek: Relfun/X - An Experimental Prolog Implementation of Relfun 48 pages

\section{D-91-02}

Jörg P. Müller: Design and Implementation of a Finite Domain Constraint Logic Programming System based on PROLOG with Coroutining 127 pages

\section{D-91-03}

Harold Boley, Klaus Elsbernd, Hans-Günther Hein, Thomas Krause: RFM Manual: Compiling RELFUN into the Relational/Functional Machine 43 pages

\section{D-91-04}

DFKI Wissenschaftlich-Technischer Jahresbericht 1990

93 Seiten

\section{D-91-06}

Gerd Kamp: Entwurf, vergleichende Beschreibung und Integration eines Arbeitsplanerstellungssystems für Drehteile

130 Seiten

D-91-07

Ansgar Bernardi, Christoph Klauck, Ralf Legleitner TEC-REP: Repräsentation von Geometrie- und Technologieinformationen

70 Seiten

D-91-08

Thomas Krause: Globale Datenflußanalyse und horizontale Compilation der relational-funktionalen Sprache RELFUN

137 Seiten

D-91-09

David Powers, Lary Reeker (Eds.):

Proceedings MLNLO'91 - Machine Learning of Natural Language and Ontology

211 pages

Note: This document is available only for a nominal charge of $25 \mathrm{DM}$ (or 15 US-\$).

\section{D-91-10}

Donald R. Steiner, Jürgen Müller (Eds.):

MAAMAW`91: Pre-Proceedings of the 3rd

European Workshop on „Modeling Autonomous

Agents and Multi-Agent Worlds“"

246 pages

Note: This document is available only for a nominal charge of $25 \mathrm{DM}$ (or $15 \mathrm{US}-\$$ ).

\section{D-91-11}

Thilo C. Horstmann:Distributed Truth Maintenance 61 pages

\section{D-91-12}

Bernd Bachmann:

$\mathrm{H}^{\text {iera }} \mathrm{C}_{\text {on }}$ - a Knowledge Representation System with Typed Hierarchies and Constraints 75 pages

\section{D-91-13}

International Workshop on Terminological Logics Organizers: Bernhard Nebel, Christof Peltason, Kai von Luck

131 pages

\section{D-91-14}

Erich Achilles, Bernhard Hollunder, Armin Laux, Jörg-Peter Mohren: KRIS : Thowledge

Representation and $\mathrm{mference}$ System

- Benutzerhandbuch -

28 Seiten

D-91-15

Harold Boley, Philipp Hanschke, Martin Harm, Knut Hinkelmann, Thomas Labisch, Manfred Meyer, Jörg Müller, Thomas Oltzen, Michael Sintek, Werner Stein, Frank Steinle:

4 CAD2NC: A Declarative Lathe-Worplanning Model Transforming CAD-like Geometries into Abstract NC Programs

100 pages

\section{D-91-16}

Jörg Thoben, Franz Schmalhofer, Thomas Reinartz: Wiederholungs-, Varianten- und Neuplanung bei der Fertigung rotationssymmetrischer Drehteile 134 Seiten

\section{D-91-17}

Andreas Becker:

Analyse der Planungsverfahren der KI im Hinblick auf ihre Eignung für die Abeitsplanung

86 Seiten

D-91-18

Thomas Reinartz: Definition von Problemklassen im Maschinenbau als eine Begriffsbildungsaufgabe 107 Seiten

D-91-19

Peter Wazinski: Objektlokalisation in graphischen Darstellungen

110 Seiten 

